This is the final peer-reviewed accepted manuscript of:

Design or not design? A numerical characterisation for piecewise Chebyshevian splines

Carolina Vittoria Beccari ${ }^{1}$, Giulio Casciola ${ }^{1}$, Marie-Laurence Mazure ${ }^{2}$

1-Department of Mathematics, University of Bologna, P.zza di Porta S.Donato 5, 40126 Bologna, Italy

2-Laboratoire Jean Kuntzmann, Université Grenoble-Alpes, BP53, Cedex 9, 38041 Grenoble, France

The final published version is available online at:

https://doi.org/10.1007/s11075-018-0533-z

Rights / License:

The terms and conditions for the reuse of this version of the manuscript are specified in the publishing policy. For all terms of use and more information see the publisher's website.

This item was downloaded from IRIS Università di Bologna (https://cris.unibo.it/)

When citing, please refer to the published version. 


\title{
Design or not design? A numerical characterisation for piecewise Chebyshevian splines
}

\author{
Carolina Vittoria Beccari · Giulio Casciola . \\ Marie-Laurence Mazure
}

\begin{abstract}
This work concerns the useful and large class of all piecewise Chebyshevian splines, in the sense of splines with pieces taken from different Extended Chebyshev spaces all of the same dimension, and with connection matrices at the knots. The subclass of those which are interesting for applications, and in particular for design, is known to be characterised by the fact that the continuity between consecutive pieces can always be controlled by identity matrices, provided that we express it by means of appropriate generalised derivatives associated with the section-spaces. Modelled on the proof of this beautiful theoretical characterisation, we provide a numerical procedure to check whether or not a given spline space lies in that subclass. Examples are given proving the usefulness of the test in situations where it is not expectable to derive exact practical conditions from the above-mentioned theoretical characterisation.
\end{abstract}

Keywords Piecewise Chebyshevian splines - Connection matrices · B-spline bases · Bernstein bases $\cdot$ (Piecewise) generalised derivatives $\cdot$ Blossoms

\section{Introduction}

Given $a<b$, take any sequence $\left(w_{0}, w_{1}, \ldots, w_{n}\right)$ of weight functions on $[a, b]$, in the sense that $w_{i}$ is both positive and $C^{n-i}$ on $[a, b]$, and, for $i=0, \ldots, n$, the associated $i$ th order linear differential operator $L_{i}$ on $C^{n}([a, b])$, obtained by alternating division by $w_{0}$, differentiation, division by $w_{1}$, differentiation, and so forth up

Carolina Vittoria Beccari

Department of Mathematics, University of Bologna, P.zza di Porta S. Donato 5, 40126 Bologna, Italy

E-mail: carolina.beccari2@unibo.it

Giulio Casciola

Department of Mathematics, University of Bologna, P.zza di Porta S. Donato 5, 40126 Bologna, Italy

E-mail: giulio.casciola@unibo.it

Marie-Laurence Mazure

Laboratoire Jean Kuntzmann, Université Grenoble-Alpes, BP53, Cedex 9, 38041 Grenoble, France

E-mail: Marie-Laurence.Mazure@univ-grenoble-alpes.fr 
to division by $w_{i}$ (referred to as generalised derivatives). It is then well-known that the $(n+1)$-dimensional space $\mathbb{E}$ composed of all functions $F \in C^{n}([a, b])$ such that $L_{n} F$ is constant on $[a, b]$ is an Extended Chebyshev space on $[a, b]$. We denote it as $\mathbb{E}=E C\left(w_{0}, w_{1}, \ldots, w_{n}\right)$. Conversely, because we are dealing with a closed bounded interval, any $(n+1)$-dimensional Extended Chebyshev space $\mathbb{E}$ on $[a, b]$ is of the form $\mathbb{E}=E C\left(w_{0}, \ldots, w_{n}\right)$, and we even know how to build all sequences of weight functions on $[a, b]$ leading to such an equality [27].

Take any sequence of knots $t_{0}<t_{1}<\cdots<t_{q}<t_{q+1}$. For each $k=0, \ldots, q$, take any sequence $\left(w_{0}^{k}, w_{1}^{k}, \ldots, w_{n}^{k}\right)$ of weight functions on $\left[t_{k}, t_{k+1}\right]$, with $w_{0}^{k}=\mathbb{I}$ (this notation stands for the constant function $\mathbb{I}(x)=1$ for all $x$ in any given interval) and denote by $L_{0}^{k}, L_{1}^{k}, \ldots, L_{n}^{k}$, the associated generalised derivatives. Select a sequence $\left(F_{0}, \ldots, F_{q}\right)$ satisfying the following two properties:

1. for each $k=0, \ldots, q, F_{k}$ belongs to $\mathbb{E}_{k}:=E C\left(\mathbb{I}, w_{1}^{k}, \ldots, w_{n}^{k}\right)$;

2. for each $k=1, \ldots, q$ and for each $i=0, \ldots, n-1, L_{i}^{k-1} F_{k-1}\left(t_{k}\right)=L_{i}^{k} F_{k}\left(t_{k}\right)$.

The continuous function $S:\left[t_{0}, t_{q+1}\right] \rightarrow \mathbb{R}$ which coincides with $F_{k}$ on $\left[t_{k}, t_{k+1}\right]$ for $k=0, \ldots, q$ is what we call a piecewise Chebyshevian spline. From the second requirement, it is well-known that, in terms of the ordinary derivatives, $S$ satisfies connection conditions

$$
\left(S\left(t_{k}\right), S^{\prime}\left(t_{k}^{+}\right), \ldots, S^{(n-1)}\left(t_{k}^{+}\right)\right)^{T}=R_{k}\left(S\left(t_{k}\right), S^{\prime}\left(t_{k}^{-}\right), \ldots, S^{(n-1)}\left(t_{k}^{-}\right)\right)^{T}, \quad k=1, \ldots, q,
$$

where each $R_{k}$ is a lower triangular matrix of order $n$ with positive diagonal entries and first column equal to $(1,0, \ldots, 0)^{T}$. It is known that

Theorem 1 The $(n+q+1)$-dimensional space $\mathbb{S}$ of all piecewise Chebyshevian spli$n e s S$ defined by the previous two properties is good for design. Conversely, any piecewise Chebyshevian spline $\mathbb{S} \subset C^{0}\left(\left[t_{0}, t_{q+1}\right]\right)$ with $t_{1}, \ldots, t_{q}$ as simple knots, supposed to be good for design, is obtained according to the previous procedure.

The expression good for design is used with the meaning of existence of refinable $B$-spline bases in $\mathbb{S}$, or, equivalently, existence of blossoms. Though stated here with a finite sequence of simple knots for convenience, Theorem 1 is more generally valid for any knot-vector, with any non-negative multiplicities [28]. Piecewise Chebyshevian splines are known to be extremely useful for design due to the great variety of shape effects they provide. Nevertheless, in practice, how can we recognise that such a given spline space, defined by its section-spaces, and connection conditions (1) is good for design? A constructive method to address this question naturally results from Theorem 1: among the infinitely many possible ways to write the section-spaces as $E C\left(\mathbb{I}, w_{1}^{k}, \ldots, w_{n}^{k}\right), k=0, \ldots, q$, can we find one so that all given connection conditions (1) can be expressed by identity matrices in terms of the associated generalised derivatives? With simple knots and $(n+1)$-dimensional section-spaces $(n \geqslant 3)$, this results in a (non-linear, except for $n=3)$ system in $(q+1) n(n-1) / 2$ unknowns and $q(n-1)$ equations, in which any $(n-2)$ consecutive connection matrices are involved at the same time, and that we have to solve positively [29,33]. For $n=3$, this provides us with an elementary necessary and sufficient condition separately on each connection matrix $R_{k}$, depending on the two corresponding section-spaces. For 
$n=4$, the complete characterisation of all good for design splines resulting from Theorem 1, already rather difficult to obtain, was achieved in [33], and exploited there in various interesting situations, see also [8]. Unless we simultaneously increase the multiplicities, the difficulty highly increases with $n$, and with simple knots, finding exact necessary and sufficient conditions is not really expectable beyond $n=4$. It is not expectable either, even in relatively low dimensions, when a significant number of knots have zero multiplicities. This is the reason why, in the present paper, we develop a numerical algorithm to replace the exact resolution of the above-mentioned system. This procedure extends and completes the one previously proposed in the special case where all interior $t_{1}, \ldots, t_{q}$ have zero multiplicities, that is, when $\mathbb{S}$ has the same dimension as each of its section-spaces [2]. In that situation, the question could equivalently be formulated as follows: is $\mathbb{S}$ an Extended Chebyshev Piecewise space good for design on $[a, b]$ relative to the interior knots $t_{1}, \ldots, t_{q}$ ?

Our numerical test for splines is built in Section 3, according to the same general guiding line as in [2] in so far as it is based on dimension diminishing via piecewise generalised derivatives. As explained in Section 2 along with all the necessary background, this decisive point is also the guiding line in the proof of the converse part of the general version of Theorem 1, see [28]. Also reminded in Section 2 is the crucial simultaneous effect of dimension diminishing on the bases functions in the spline space and in the section-spaces which plays a prominent role for the test and highly simplifies it. In Section 4, we address several situations making the usefulness of the numerical test clear.

\section{Context and background}

This section aims at recalling the crucial results which the present work relies on. Our overview will be as brief as possible. For further acquaintance with Extended Chebyshev spaces, see, for instance, [15,37, 18,35,24,25,22,10,27], with Chebyshevian splines, see [37,35,3,4], with piecewise Chebyshevian splines, see [1,20,23, 11,22, $26,28]$ and additional references therein.

\subsection{Extended Chebyshev spaces}

Throughout this subsection we work on a given closed bounded interval $[a, b], a<b$. Given an integer $n \geqslant 0$, an $(n+1)$-linear space $\mathbb{E} \subset C^{n}([a, b])$ is said to be an Extended Chebyshev space on $[a, b]$ (for short, EC-space on $[a, b]$ ) if any non-zero $F \in \mathbb{E}$ vanishes at most $n$ times on $[a, b]$, counting multiplicities up to $(n+1)$. Equivalently, $\mathbb{E}$ is an EC-space on $[a, b]$ if any Hermite interpolation problem in $(n+1)$ data in $[a, b]$ is unisolvent in $\mathbb{E}$.

Bases of the Bernstein-type play a prominent role in EC-spaces, and we remind their definition below.

Definition 1 A sequence $\left(V_{0}, \ldots, V_{n}\right)$ in $C^{n}([a, b])$ is said to be a Bernstein-like basis relative to $(a, b)$ if, for each $k=0, \ldots, n, V_{k}$ vanishes exactly $k$ times at $a$, and exactly $(n-k)$ times at $b$, and is positive on $] a, b\left[\right.$. A sequence $\left(B_{0}, \ldots, B_{n}\right)$ in $C^{n}([a, b])$ is 
said to be a Bernstein basis relative to $(a, b)$ if it is a Bernstein-like basis relative to $(a, b)$ which is normalised, in the sense that it satisfies $\sum_{i=0}^{n} B_{i}=\mathbb{I}$.

Any EC-space on $[a, b]$ possesses infinitely many different Bernstein-like bases relative to $(a, b)$. Clearly, for an EC-space on $[a, b]$ to possess a Bernstein basis relative to $(a, b)$ it is necessary that it contains the constants. Nevertheless, this is not sufficient, unless its dimension is at most two. Below we state the first key-point for the present work. [24,27].

Theorem 2 Given an integer $n \geqslant 1$, let $\mathbb{E}$ be an $(n+1)$-dimensional EC-space on $[a, b]$, supposed to contain the constants, the following properties are then equivalent:

1. E possesses a Bernstein basis relative to $(a, b)$;

2. the space $D \mathbb{E}:=\left\{D F:=F^{\prime} \mid F \in \mathbb{E}\right\}$ is an (n-dimensional) EC-space on $[a, b]$;

3. blossoms exist in $\mathbb{E}$.

Furthermore, if any of these properties is satisfied, then it is possible to develop all the classical geometric design algorithms in $\mathbb{E}$, and the Bernstein basis relative to $(a, b)$ is the optimal normalised totally positive basis in $\mathbb{E}$.

Though we will avoid explicitly using blossoms as far as possible, they are implicitly involved in all results reminded in this subsection, and their properties are essential in the proof of Theorem 2. This is the reason why we mentioned them. As for the expression optimal normalised totally positive basis, the reader can simply understand it as the best possible basis for design. For the precise meaning, see [22], and also $[14,9]$. Theorem 2 justifies the definition below.

Definition 2 A given $(n+1)$-dimensional EC-space $\mathbb{E}$ on $[a, b]$ is said to be good for design on $[a, b]$ when it possesses a Bernstein basis relative to $(a, b)$ (or, equivalently, when it contains the constants and possesses blossoms).

The second key-point needed is closely connected with the proof of Theorem 2 (see [27]).

Theorem 3 Let $\mathbb{E}$ be an $(n+1)$-dimensional EC-space on $[a, b]$, and let $\left(V_{0}, \ldots, V_{n}\right)$ be a Bernstein-like basis relative to $(a, b)$ in $\mathbb{E}$. Then, for a function $w_{0} \in \mathbb{E}$, expanded as $w_{0}=\sum_{i=0}^{n} \alpha_{i} V_{i}$, the following properties are equivalent:

1. $\alpha_{0}, \alpha_{1}, \ldots, \alpha_{n}$ are all positive;

2. $w_{0}$ is positive on $[a, b]$ and, setting $L_{0} V:=V / w_{0}$ for all functions $V$ defined on $[a, b]$, the space $L_{0} \mathbb{E}$ is good for design (i.e., if $n \geqslant 1, D L_{0} \mathbb{E}$ is an EC-space on $[a, b])$.

Remark 1 In connection with Theorems 2 and 3, the recursive passage from Bernstein bases / Bernstein-like bases to Bernstein-like bases / Bernstein bases, respectively, will be crucial for the the numerical test built in next section. We therefore have to recall it subsequently. Let us start with an $(n+1)$-dimensional EC-space good for design on $[a, b], \mathbb{E}$, and its Bernstein basis $\left(B_{0}, \ldots, B_{n}\right)$ relative to $(a, b)$. 
- From $\mathbb{E}$ to $D \mathbb{E}:$ Consider the functions ${ }^{1}$

$$
B_{i}^{\star}:=\sum_{k=i}^{n} B_{k}=\mathbb{I}-\sum_{k=0}^{i-1} B_{k}, \quad i=0, \ldots, n,
$$

so that, in particular,

$$
B_{0}^{\star}=\mathbb{I}, \quad B_{n}^{\star}=B_{n}, \quad B_{i}=B_{i}^{\star}-B_{i+1}^{\star} \text { for } i=0, \ldots, n,
$$

with the convention that $B_{n+1}^{\star}=0$. Moreover, from (2) we can see that, for each $i=1, \ldots, n$, the function $B_{i}^{\star}$ vanishes exactly $i$ times at $a$, while the function $\mathbb{I}-B_{i}^{\star}$ vanishes exactly $(n-i+1)$ times at $b$. Setting [24]

$$
V_{i}:=D B_{i+1}^{\star}=\sum_{k=i+1}^{n} D B_{k}=-\sum_{k=0}^{i} D B_{k}, \quad i=0, \ldots, n-1,
$$

we can see that, for $i=0, \ldots, n-1$, the function $V_{i}$ vanishes exactly $i$ times at $a$ and exactly $(n-1-i)$ times at $b$. It is also positive close to $a$ due to the equality $V_{i}^{(i)}(a)=$ $B_{i+1}{ }^{(i+1)}(a)$. Since, according to Theorem $2, D \mathbb{E}$ is an EC-space on $[a, b]$, we can thus conclude that that $\left(V_{0}, \ldots, V_{n-1}\right)$ is a Bernstein-like basis relative to $(a, b)$. Moreover, expansions in that basis can easily be derived from expansions in the Bernstein basis in $\mathbb{E}$. Indeed, given any real numbers $\beta_{0}, \ldots, \beta_{n}$

$$
F:=\sum_{i=0}^{n} \beta_{i} B_{i} \quad \Longrightarrow \quad D F:=\sum_{i=0}^{n-1}\left(\beta_{i+1}-\beta_{i}\right) V_{i} .
$$

- From $D \mathbb{E}$ to $L_{1} \mathbb{E}$ : Consider a function

$$
w_{1}:=\sum_{i=0}^{n-1} \alpha_{i} V_{i}, \quad \text { with positive } \alpha_{0}, \ldots, \alpha_{n-1} .
$$

Out of division of the two hand-sides of (6) by the positive function $w_{1}$, we obtain

$$
\mathbb{I}=\sum_{i=0}^{n-1} \bar{B}_{i}, \quad \text { with } \bar{B}_{i}:=\frac{\alpha_{i} V_{i}}{w_{1}} \text { for } i=0, \ldots, n-1 .
$$

Clearly, $\left(\bar{B}_{0}, \ldots, \bar{B}_{n-1}\right)$ is the Bernstein basis relative to $(a, b)$ in $L_{1} \mathbb{E}:=\frac{1}{w_{1}} D \mathbb{E}$ which is an $n$-dimensional EC-space good for design on $[a, b]$ (see Theorem 3).

This is one step of dimension diminishing within the class of all EC-spaces good for design on $[a, b]$. Starting from $L_{1} \mathbb{E}$ and its Bernstein basis relative to $(a, b)$, we can iterate the process. This alternation between differentiation and division by appropriate positive functions provides us with all ways to write $\mathbb{E}$ as $\mathbb{E}=E C\left(\mathbb{I}, w_{1}, \ldots, w_{n}\right)$, see [27]. This dimension diminishing process can be understood as alternating between design (that is, successively in $\mathbb{E}, L_{1} \mathbb{E}, \ldots$, which are EC-spaces good for design on $[a, b]$ ) and interpolation (that is, successively in $D \mathbb{E}, D L_{1} \mathbb{E}, \ldots$, which are EC-spaces on $[a, b])$.

\footnotetext{
1 It should be observed that, when $\mathbb{E}$ is the degree $n$ polynomial space, these $B_{i}^{\star}, i=0, \ldots, n$, are the functions initially introduced by Pierre Bézier, under the pseudonym Onésime Durand, before realising that their differences (Bernstein polynomials) were easier to manipulate for design. See [17] and references to P. Bézier therein.
} 
2.2 Piecewise Chebyshevian splines

From now on, we consider a fixed bi-infinite sequence $\mathbb{T}$ of knots $t_{k}, k \in \mathbb{Z}$, with $t_{k}<t_{k+1}$ for all $k$, and the associated interval $\left.I:=\right] \inf _{k} t_{k}, \sup _{k} t_{k}[$. In general, we will not deal with functions on $I$ but with piecewise functions $F$ on $(\mathrm{I} ; \mathbb{T})$, defined on $\cup_{k \in \mathbb{Z}}\left[t_{k}^{+}, t_{k+1}^{-}\right]$, that is, separately on each $\left[t_{k}^{+}, t_{k+1}^{-}\right]$, with possibly $F\left(t_{k}^{+}\right) \neq F\left(t_{k}^{-}\right)$. In this context, equality, differentiation, positivity, and so forth, will be defined piecewisely. Each $t_{k}$ is allocated a non-negative multiplicity $m_{k}$, with

$$
\sum_{i \leqslant 0} m_{i}=\sum_{i \geqslant 0} m_{i}=+\infty .
$$

Due to this requirement, the extended knot-vector $\mathbb{K}:=\left(t_{k}{ }^{\left[m_{k}\right]}\right)_{k \in \mathbb{Z}}$ formed by the knots repeated with their multiplicities can be written as a bi-infinite sequence

$$
\mathbb{K}=\left(\xi_{\ell}\right)_{\ell \in \mathbb{Z}}, \quad \text { with } \xi_{\ell} \leqslant \xi_{\ell+1} .
$$

To define a spline space we additionally consider:

- a bi-infinite sequence of section spaces $\mathbb{E}_{k}, k \in \mathbb{Z}$ : each $\mathbb{E}_{k}$ is an $(n+1)$-dimensional EC-space on $\left[t_{k}, t_{k+1}\right]$;

- a bi-infinite sequence of connection matrices $R_{k}, k \in \mathbb{Z}$ : each $R_{k}$ is a lower triangular matrix of order $\left(n-m_{k}+1\right)$ with positive diagonal entries.

Based on these data, a piecewise Chebyshevian spline (in short, PEC-spline) is a piecewise function $S$ on $(\mathrm{I} ; \mathbb{T})$ meeting the following two requirements:

1 - for each $k \in \mathbb{Z}$, the restriction of $S$ to $\left[t_{k}^{+}, t_{k+1}^{-}\right]$coincides with an element of the section-space $\mathbb{E}_{k}$;

2- $S$ satisfies the connection conditions:

$$
\left(S\left(t_{k}^{+}\right), S^{\prime}\left(t_{k}^{+}\right), \ldots, S^{\left(n-m_{k}\right)}\left(t_{k}^{+}\right)\right)^{T}=R_{k}\left(S\left(t_{k}^{-}\right), S^{\prime}\left(t_{k}^{-}\right) \ldots, S^{\left(n-m_{k}\right)}\left(t_{k}^{-}\right)\right)^{T}, \quad k \in \mathbb{Z} .
$$

Subsequently we denote by $\mathbb{S}$ the set all such splines. At a knot $t_{k}$, there are two reasons why a spline $S \in \mathbb{S}$ can be discontinuous. Either $m_{k} \geqslant n+1$ and there is no relation between $t_{k}^{-}$and $t_{k}^{+}$; or $m_{k} \leqslant n$ and the first diagonal entry of the connection matrix $R_{k}$ is not equal to 1 . Observe that piecewise multiplication by some piecewise function $\omega$ which is piecewise positive and piecewise $C^{n}$ on $(I ; \mathbb{T})$ transforms $\mathbb{S}$ in another PEC-spline space in which, at each knot $t_{k}$, the new connection matrix can be calculated from $R_{k}$ and $\omega$ (see, for instance, [28]). Integration also transforms a PEC-spline space into another one which contains the constants.

Denote by $\Lambda_{n}(\mathbb{K})$ the set of all integers $\ell$ such that $\xi_{\ell}<\xi_{\ell+n+1}$.

Definition 3 A sequence $Q_{\ell} \in \mathbb{S}, \ell \in \Lambda_{n}(\mathbb{K})$, is a $B$-spline-like basis if it meets the usual requirements below:

- support property: for each $\ell \in \Lambda_{n}(\mathbb{K}), Q_{\ell}$ is piecewise zero outside $\left[\xi_{\ell}, \xi_{\ell+n+1}\right]$;

- positivity property: for each $\ell \in \Lambda_{n}(\mathbb{K}), Q_{\ell}$ is piecewise positive on $] \xi_{\ell}, \xi_{\ell+n+1}[$;

- endpoint property: for each $\ell \in \Lambda_{n}(\mathbb{K}), Q_{\ell}$ vanishes exactly $(n-s+1)$ times at $\xi_{\ell}^{+}$ and exactly $\left(n-s^{\prime}+1\right)$ at $\xi_{\ell+n+1}^{-}$, where $s:=\#\left\{j \geqslant \ell \mid \xi_{j}=\xi_{\ell}\right\}$ and $s^{\prime}:=\#\{j \leqslant$ $\left.\ell+n+1 \mid \xi_{j}=\xi_{\ell+n+1}\right\}$. 
A sequence $N_{\ell} \in \mathbb{S}, \ell \in \Lambda_{n}(\mathbb{K})$, is a $B$-spline basis if it is a B-spline-like basis which is normalised, in the sense that $\sum_{\ell \in \Lambda_{n}(\mathbb{K})} N_{\ell}(x)=1$ for all $x \in \cup_{k \in \mathbb{Z}}\left[t_{k}^{+}, t_{k+1}^{-}\right]$.

Subsequently, for the sake of simplicity, given a B-spline-like basis $Q_{\ell} \in \mathbb{S}, \ell \in$ $\Lambda_{n}(\mathbb{K})$, we will abusively write $S=\sum_{\ell} \alpha_{\ell} Q_{\ell}$ with the meaning of the following pointwise equality:

$$
S(x)=\sum_{\ell \in \Lambda_{n}(\mathbb{K})} \alpha_{\ell} Q_{\ell}(x) \quad \text { for all } x \in\left[t_{k}^{+}, t_{k+1}^{-}\right] \text {and all } k \in \mathbb{Z} .
$$

For instance, with this (double) abuse of notations, the normalisation property of a Bspline basis $N_{\ell}, \ell \in \Lambda_{n}(\mathbb{K})$, can be written as $\sum_{\ell} N_{\ell}=\mathbb{I}$, but the reader should keep in mind first that it is a piecewise and pointwise equality, second that it may not concern all integers.

The spline space $\mathbb{S}$ contains the constant function $\mathbb{I}$ if and only if, firstly each section-space contains the constants, and secondly, for each $k$ such that $m_{k} \leqslant n$, the first column of the connection matrix $R_{k}$ is equal to $(1,0, \ldots, 0)^{T}$. If $\mathbb{S}$ contains the constants and $n \geqslant 1$, we can assert that the space $D \mathbb{S}$ obtained by piecewise differentiation is a PEC-spline space only if, for each $k, D \mathbb{E}_{k}$ is an EC-space on $\left[t_{k}, t_{k+1}\right]$, that is, only if the section-spaces $\mathbb{E}_{k}$ are EC-spaces good for design on their intervals. A spline version of Theorem 2 can be stated as follows (see [23,22] and other useful references therein).

Theorem 4 Assume the PEC-spline space $\mathbb{S}$ to contain the constants. Then, the following three properties are equivalent:

1. $\mathbb{S}$ possesses a B-spline basis, and so does any spline space obtained from $\mathbb{S}$ by knot insertion;

2. the space DS is a PEC-spline space, it possesses B-spline-like bases, and so does any spline space obtained from $D \mathbb{S}$ by knot insertion;

3. blossoms exist in $\mathbb{S}$.

Furthermore, if one of these properties is satisfied, then it is possible to develop all the classical geometric design algorithms for splines in $\mathbb{S}$, and its B-spline basis is totally positive.

Obviously, the second property above is meaningful only if $n \geqslant 1$. As in the nonspline case, Theorem 4 highly justifies the following definition.

Definition 4 A PEC-spline space $\mathbb{S}$ being given, we say that $\mathbb{S}$ is good for design if it possesses a B-spline basis and so does any spline space derived from $\mathbb{S}$ by insertion of knots (or, equivalently, if $\mathbb{S}$ contains the constants and possesses blossoms).

Clearly, all section-spaces of a PEC-spline space $\mathbb{S}$ good for design are themselves good for design on their own intervals. From the study carried out in [32], it follows that condition 2 of Theorem 4 is satisfied if and only if the restriction of $D \mathbb{S}$ to any closed bounded interval ensures unisolvence of all corresponding Hermite (left/right) interpolation problems under the classical Schoenberg-Whitney conditions and the same holds true for after knot insertion. It is convenient to introduce the following definition. 
Definition 5 A PEC-spline space $\mathbb{S}$ being given, we say that $\mathbb{S}$ is good for interpolation if it possesses B-spline-like bases and so does any spline space derived from $\mathbb{S}$ by insertion of knots.

According to the latter two definitions, a PEC-spline space which is good for design is good for interpolation. Moreover, Theorem 4 can now be reformulated as follows.

Theorem 5 Given PEC-spline space $\mathbb{S}$, let $\widehat{\mathbb{S}}$ denote the PEC-spline space (containing the constants) obtained by continuous integration. The following two properties are equivalent:

1. S is good for interpolation;

2. $\widehat{\mathbb{S}}$ is good for design.

Assume that a PEC-spline space $\mathbb{S}$ possesses a spline $w_{0}$ which is positive on $(\mathrm{I} ; \mathbb{T})$. Then we can consider the piecewise division $L_{0}$ by $w_{0}$, that is, $L_{0} V:=\frac{V}{w_{0}}$ for all piecewise functions $V$ on $(\mathrm{I} ; \mathbb{T})$. The space $L_{0} \mathbb{S}$ is a PEC-space on $(\mathrm{I} ; \mathbb{T})$, in which the connection matrices can be calculated from $w_{0}$ and the connection matrices in $\mathbb{S}$. Moreover $L_{0} \mathbb{S}$ contains the constants. Still, even if $\mathbb{S}$ possesses a B-spline-like basis, this does not guarantee that $L_{0} \mathbb{S}$ possesses a B-spline basis. The spline version of Theorem 3 is recalled below.

Theorem 6 [28] Assume the PEC-spline space $\mathbb{S}$ to be good for interpolation, and let $Q_{\ell}, \ell \in \Lambda_{n}(\mathbb{K})$, be a $B$-spline-like basis in $\mathbb{S}$. Then, for a spline $w_{0} \in \mathbb{S}$, expanded as $w_{0}=\sum_{\ell} \alpha_{\ell} Q_{\ell}$, the following properties are equivalent:

1. all coefficients $\alpha_{\ell}$ are positive;

2. $w_{0}$ is piecewise positive on $(I ; \mathbb{T})$ and, setting $L_{0} V:=V / w_{0}$ for all piecewise functions on $(\mathrm{I} ; \mathbb{T})$ (piecewise division by $w_{0}$ ), the PEC-spline space $L_{0} \mathbb{S}$ is good for design.

Remark 2 Similarly to what we mentioned in Remark 1, the recursive passage from B-spline / B-spline-like bases to B-spline-like / B-spline bases will be crucial for the the numerical test built in next section. Here, we assume that $\mathbb{S}$ is a PEC-spline space good for design on $(\mathrm{I} ; \mathbb{T})$, with $(n+1)$-dimensional section-spaces, $n \geqslant 1$, and we denote by $N_{\ell}, \ell \in \Lambda_{n}(\mathbb{K})$, its B-spline basis.

- From $\mathbb{S}$ to $D \mathbb{S}$ : Consider the splines $\mathbf{N}_{\ell}^{\star} \in \mathbb{S}$ defined by ${ }^{2}$

$$
N_{\ell}^{\star}:=\sum_{p \geqslant \ell} N_{p}=\mathbb{I}-\sum_{p \leqslant \ell-1} N_{p}, \quad \ell \in \Lambda_{n}(\mathbb{K}) .
$$

so that, in particular,

$$
N_{\ell}=N_{\ell}^{\star}-N_{\ell+1}^{\star}, \quad \ell \in \Lambda_{n}(\mathbb{K}) .
$$

When it is defined, the spline $N_{\ell}^{\star}$ is piecewise zero (resp. one) on the left (resp. on the right) of $\left[\xi_{\ell}^{+}, \xi_{\ell+n}^{-}\right]$. In particular if $\xi_{\ell}=\xi_{\ell+n}$, it is piecewise constant. Otherwise, $\ell \in \Lambda_{n-1}(\mathbb{K})$, and from (11) we can see that $N_{\ell}^{\star}$ vanishes exactly as many times as

\footnotetext{
2 In the polynomial spline setting, this corresponds to the transition functions by L. Schumaker, p. 141 of [37].
} 
$N_{\ell}$ at $\xi_{\ell}^{+}$, while the spline $\mathbb{I}-N_{\ell}^{\star}$ vanishes exactly as many times as $N_{\ell-1}$ at $\xi_{\ell+n}^{-}$. Setting

$$
Q_{\ell}:=D N_{\ell}^{\star}, \quad \ell \in \Lambda_{n-1}(\mathbb{K}),
$$

for each $\ell \in \Lambda_{n-1}(\mathbb{K})$, the piecewise function $Q_{\ell}$ vanishes exactly $(n-s)$ times at $\xi_{\ell}$ and exactly $\left(n-s^{\prime}\right)$ times at $\xi_{\ell+n}$, where $s$ and $s^{\prime}$ are defined in the endpoint property of Definition 3. It is also positive close to $\xi_{\ell}^{+}$due to the equality $Q_{\ell}^{(n-s-1)}\left(\xi_{\ell}^{+}\right)=$ $N_{\ell}^{(n-s+1)}\left(\xi_{\ell}^{+}\right)$. Since, according to Theorem $5, D \mathbb{S}$ is a PEC-spline space good for interpolation on $(\mathrm{I} ; \mathbb{T})$, we can thus conclude that the sequence $Q_{\ell}, \ell \in \Lambda_{n-1}(\mathbb{K})$, forms a B-spline-like basis in $D \mathbb{S}$. Moreover, expansions in that basis can easily be derived from expansions in the B-spline basis. Indeed, given any spline $S \in \mathbb{S}$,

$$
S=\sum_{\ell} \alpha_{\ell} N_{\ell} \quad \Longrightarrow \quad D S:=\sum_{\ell}\left(\alpha_{\ell}-\alpha_{\ell-1}\right) Q_{\ell} .
$$

- From $D \mathbb{S}$ to $L_{1} \mathbb{S}$ : Consider a spline $w_{1} \in D \mathbb{S}$, given by

$$
w_{1}:=\sum_{\ell} \alpha_{\ell} Q_{\ell}, \quad \text { with positive } \alpha_{\ell} \text {. }
$$

This spline $w_{1}$ is piecewise positive on $(\mathrm{I} ; \mathbb{T})$ and piecewise division of both handsides of (15) by $w_{1}$ yields the piecewise equality

$$
\mathbb{I}=\sum_{\ell} \bar{N}_{\ell}, \quad \text { with } \bar{N}_{\ell}:=\frac{\alpha_{\ell} Q_{\ell}}{w_{1}} \text { for } \ell \in \Lambda_{n-1}(\mathbb{K}) .
$$

The sequence $\bar{N}_{\ell}, \ell \in \Lambda_{n-1}(\mathbb{K})$, is the B-spline basis in $L_{1} \mathbb{S}:=\frac{1}{w_{1}} D \mathbb{S}$ which is a PECspline space good for design on $(\mathrm{I} ; \mathbb{T})$, with $n$-dimensional section-spaces, and with connection matrices deduced from those in $\mathbb{S}$ and from (15).

Above we have described one step of dimension diminishing within the class of all PEC-spline spaces good for design on $(I ; \mathbb{T})$, obtained through the piecewise generalised derivative $L_{1}$. Observe that, for all $S \in \mathbb{S}$, the function $L_{1} S$ is continuous at each knot $t_{k}$ for which $m_{k} \leqslant n-1$. Starting from $L_{1} \mathbb{S}$ and its B-spline basis we can iterate the process. This alternation between (left/right) differentiation and piecewise division by appropriate positive piecewise functions on $(\mathrm{I} ; \mathbb{T})$ provides us with all local systems of generalised derivatives relative to which the connection conditions in $\mathbb{S}$ are expressed by identity matrices (see the general version of Theorem 1 in [28]). According to Theorem 5, this dimension diminishing process produces alternation between PEC-spline spaces good for design $\left(\mathbb{S}, L_{1} \mathbb{S}\right.$, etc) and PEC-spline spaces good for interpolation $\left(D \mathbb{S}, D L_{1} \mathbb{S}\right.$, etc).

Remark 3 Even if we require that all multiplicities be bounded above by $n$ in the initial space (continuous splines), it may be the case that after a few steps we will be working in a discontinuous PEC-space $L_{r} \mathbb{S}$, in which the equality $\Lambda_{n-r}(\mathbb{K})=\mathbb{Z}$ will no longer be valid. Then, one can either continue with the convention introduced earlier, or, if we want to avoid this difficulty, split the space $L_{r} \mathbb{S}$ into several PEC-spline spaces of the form $L_{r} \mathbb{S}_{k}^{-}, L_{r} \mathbb{S}_{k}^{+}$, or $L_{r} \mathbb{S}_{k, k^{\prime}}$, obtained by restriction to $\cup_{j \leqslant k-1}\left[t_{j}^{+}, t_{j+1}^{-}\right]$, $\cup_{j \geqslant k}\left[t_{j}^{+}, t_{j+1}^{-}\right], \cup_{k \leqslant j \leqslant k^{\prime}-1}\left[t_{j}^{+}, t_{j+1}^{-}\right]$, with $k<k^{\prime}$, respectively, in which all interior multiplicities will be bounded above in an appropriate way. 
2.3 Towards the numerical test

Subsequently, we present some further arguments on which our test will be closely modelled.

\subsubsection{Positivity property of local expansions}

In this subsection, we consider local properties of the B-spline (-like) bases. For convenience, we assume $m_{k} \leqslant n$ for all $k \in \mathbb{Z}$, and consider a PEC-spline space $\mathbb{S}$ with $(n+1)$-dimensional section-spaces.

Proposition 1 Let $\mathbb{S}$ be a PEC-spline space good for design, and let $N_{\ell}, \ell \in \mathbb{Z}$, be the $B$-spline basis in $\mathbb{S}$. For any integers $\ell, k \in \mathbb{Z}$, we denote by $N_{\ell}^{k}$ the restriction to $\left[t_{k}, t_{k+1}\right]$ of the $B$-spline $N_{\ell}$, and by $\alpha_{\ell, k, i}$ its Bézier points relative to $\left(t_{k}, t_{k+1}\right)$, that is,

$$
N_{\ell}^{k}=\sum_{i=0}^{n} \alpha_{\ell, k, i} B_{k, i}, \quad k, \ell \in \mathbb{Z}
$$

Then, for $\left[t_{k}, t_{k+1}\right] \subset\left[\xi_{\ell}, \xi_{\ell+n+1}\right]$, all $\alpha_{\ell, k, i}$ 's are positive except in accordance with the end point property, that is,

$$
\begin{aligned}
& \text { if } t_{k}=\xi_{\ell}, \text { then } \alpha_{\ell, k, 0}=\alpha_{\ell, k, 1}=\cdots=\alpha_{\ell, k, n-s}=0 \\
& \text { and } \alpha_{\ell, k, i}>0 \text { for } i=n-s+1, \ldots, n, \\
& \text { if } \left.\left[t_{k}, t_{k+1}\right] \subset\right] \xi_{\ell}, \xi_{\ell+n+1}\left[, \text { then } \alpha_{\ell, k, i}>0 \text { for } i=0, \ldots, n,\right. \\
& \text { if } t_{k+1}=\xi_{\ell+n+1}, \text { then } \alpha_{\ell, k, i}>0 \text { for } i=0, \ldots, s^{\prime}-1 \\
& \text { and } \alpha_{\ell, k, s^{\prime}}=\alpha_{\ell, k, s^{\prime}+1}=\cdots=\alpha_{\ell, k, n}=0,
\end{aligned}
$$

the integers $s, s^{\prime}$ being those introduced in Definition 3.

Proof The poles of a spline $S \in \mathbb{S}$ are the real numbers $\alpha_{\ell}$ such that, with the convention adopted earlier, $S=\sum_{j} \alpha_{j} N_{j}$. As is classical with polynomials / polynomial splines [36], they can be expressed as

$$
\alpha_{j}:=s\left(\xi_{j+1}, \ldots, \xi_{j+n}\right) \in \mathbb{R}^{d}, \quad j \in \mathbb{Z},
$$

where $s$ is the blossom of $S$, defined on the set of all $n$-tuples $\left(x_{1}, \ldots, x_{n}\right)$ which are admissible with respect to the knot-vector $\mathbb{K}$, in the sense that, up to permutation, they can be written as follows

$$
\left.\left(x_{1}, \ldots, x_{n}\right)=\left(\xi_{\ell_{1}+1}, \xi_{\ell_{1}+2}, \ldots, \xi_{\ell_{2}}, y_{1}, \ldots, y_{p}\right), \quad \text { with } y_{1}, \ldots, y_{p} \in\right] \xi_{\ell_{1}}, \xi_{\ell_{2}+1}[\text {. }
$$

Classically, all values of the blossom $s$ of $S$ can be computed from the poles of $S$ by means of a corner-cutting evaluation algorithm. Indeed, supposing that (19) holds, the fundamental properties of blossoms (see for instance [28]) guarantee that $s\left(x_{1}, \ldots, x_{n}\right)$ is a strictly convex combination of the $(p+1)$ poles $\alpha_{\ell_{2}-n}, \ldots, \alpha_{\ell_{1}}$ of $S$, with coefficients independent of $S$. Given $k \in \mathbb{Z}$, this applies in particular to all $n$-tuples of the form

$$
\left(x_{1}, \ldots, x_{n}\right)=\left(t_{k}^{[n-i]}, t_{k+1}^{[i]}\right), \quad i=0, \ldots, n,
$$


where the notation $x^{[r]}$ stands for $x$ repeated $r$ times. The real numbers $s\left(t_{k}{ }^{[n-i]}, t_{k+1}{ }^{[i]}\right)$, $i=0, \ldots, n$, are the Bézier points (relative to $\left(t_{k}, t_{k+1}\right)$ ) of the function $F_{k} \in \mathbb{E}_{k}$ which coincides with $S$ on $\left[t_{k}, t_{k+1}\right]$. This means that

$$
F_{k}=\sum_{i=0}^{n} s\left(t_{k}^{[n-i]}, t_{k+1}^{[i]}\right) B_{k, i}
$$

Apply the evaluation algorithm when $S$ is the B-spline $N_{\ell}$, for some $\ell \in \mathbb{Z}$. In that case, we work with the poles

$$
s\left(\xi_{j+1}, \ldots, \xi_{j+n}\right)=1 \text { if } j=\ell, \quad s\left(\xi_{j+1}, \ldots, \xi_{j+n}\right)=0 \text { otherwise }
$$

Without going more into details, this yields the claimed result.

We refer to (18) as the local expansion positivity property of the B-spline basis. As a consequence, a similar property can be stated when $\mathbb{S}$ is good for interpolation.

Corollary 1 Assume that $\mathbb{S}$ be a PEC-spline space good for interpolation, and let $Q_{\ell}, \ell \in \mathbb{Z}$, be any $B$-spline like basis in $\mathbb{S}$. For any integers $\ell, k \in \mathbb{Z}$, we denote by $Q_{\ell}^{k}$ the restriction to $\left[t_{k}^{+}, t_{k+1}^{-}\right]$of the B-spline-like $Q_{\ell}$, and by $\gamma_{\ell, k, i}$ the coefficients of its expansion in a Bernstein-like basis $\left(V_{k, 0}, \ldots, V_{k, n}\right)$ relative to $\left(t_{k}, t_{k+1}\right)$ in the section-space $\mathbb{E}_{k}$, that is,

$$
Q_{\ell}^{k}=\sum_{i=0}^{n} \gamma_{\ell, k, i} V_{k, i}, \quad k, \ell \in \mathbb{Z} .
$$

Then, these local expansions satisfy the positivity property (18).

Proof Take any positive numbers $\alpha_{\ell}, \ell \in \mathbb{Z}$, and the associated piecewise positive spline $w_{0}=\sum_{\ell} \alpha_{\ell} Q_{\ell} \in \mathbb{S}$. If $L_{0}$ denotes the piecewise division by $w_{0}$, we know that the PEC-spline space $L_{0} \mathbb{S}$ is good for design (Theorem 6), its B-spline basis $N_{\ell}, \ell \in \mathbb{Z}$, being obtained as follows:

$$
N_{\ell}:=\frac{\alpha_{\ell} Q_{\ell}}{w_{0}}, \quad \ell \in \mathbb{Z}
$$

Denote its local expansions in the Bernstein bases $\left(B_{k, 0}, \ldots, B_{k, n}\right)$ of the sectionspaces in $L_{0} \mathbb{S}$ as in (17). They satisfy the positivity property (18). If $w_{0}^{k}$ is the restriction of $w_{0}$ to $\left[t_{k}^{+}, t_{k+1}^{-}\right]$, we obtain

$$
Q_{\ell}^{k}=\sum_{i=0}^{n} \alpha_{\ell, k, i} \frac{w_{0}^{k}}{\alpha_{\ell}} B_{k, i} .
$$

Given that the sequence $\left(\frac{w_{0}^{k}}{\alpha_{\ell}} B_{k, 0}, \ldots, \frac{w_{0}^{k}}{\alpha_{\ell}} B_{k, n}\right)$ is also a Bernstein-like basis relative to $\left(t_{k}, t_{k+1}\right)$ in the section-space $\mathbb{E}_{k}$, the claimed result is proved. 


\subsubsection{One specific dimension diminishing process}

Here, the PEC-spline space $\mathbb{S}$ is assumed to be good for design. We have seen that there are infinitely many different ways to diminish the dimension of the sectionspaces while keeping within the class of all PEC-spline spaces good for design. We will now describe the simplest possible process, obtained by choosing all coefficients $\alpha_{\ell}$ in (15) to be equal to 1 , and repeating this at each step. We thus take

$$
w_{1}:=\sum_{\ell} Q_{\ell}
$$

the B-spline-like basis $Q_{\ell}, \ell \in \mathbb{Z}$, in the PEC-spline space $D \mathbb{S}$ being defined by (13). In the PEC-spline space good for design $L_{1} \mathbb{S}$, the $\mathrm{B}$-spline basis is then defined piecewisely by

$$
\bar{N}_{\ell}:=\frac{Q_{\ell}}{w_{1}}, \quad \ell \in \mathbb{Z}
$$

Repeating the process, the B-spline-like basis in the PEC-spline space $D L_{1} \mathbb{S}$ is defined by

$$
\bar{Q}_{\ell}:=D \bar{N}_{\ell}^{\star}, \quad \ell \in \Lambda_{n-2}(\mathbb{K}), \quad \text { with } \bar{N}_{\ell}^{\star}:=\sum_{p \geqslant \ell} \bar{N}_{p}
$$

We want to calculate the local expansions of this new B-spline-like basis in function of the local expansions of the B-spline-like basis at the previous level. With this in view, let us first see how the previous steps in $\mathbb{S}$ operate on the section-spaces. In $D \mathbb{E}_{k}$, we take the Bernstein-like basis $\left(V_{k, 0}, \ldots, V_{k, n-1}\right)$ relative to $\left(t_{k}, t_{k+1}\right)$ defined by

$$
V_{k, i}:=D B_{k, i+1}^{\star} \text { for } i=0, \ldots, n-1, \quad \text { where } B_{k, i}^{\star}:=\sum_{r=i}^{n} B_{k, r} \text { for } i=0, \ldots, n .
$$

Then, consider the expansion

$$
w_{1}^{k}=\sum_{i=0}^{n-1} \delta_{k, i} V_{k, i}
$$

in which we know that all coefficients $\delta_{k, i}$ are positive because the space $L_{1}^{k} \mathbb{E}_{k}:=$ $1 / w_{1}^{k} D \mathbb{E}_{k}$ is an EC-space good for design on $\left[t_{k}, t_{k+1}\right]$ (Theorem 3 ). In $L_{1}^{k} \mathbb{E}_{k}$, the Bernstein basis $\left(\bar{B}_{k, 0}, \ldots, \bar{B}_{k, n-1}\right)$ is given by

$$
\bar{B}_{k, i}:=\frac{\delta_{k, i} V_{k, i}}{w_{1}^{k}}, \quad i=0, \ldots, n-1 .
$$

At the next step, in $D L_{1}^{k} \mathbb{E}_{k}$ we take the Bernstein-like basis $\left(\bar{V}_{k, 0}, \ldots, \bar{V}_{k, n-2}\right)$ obtained by

$$
\bar{V}_{k, i}:=D \bar{B}_{k, i+1}^{\star} \text { for } i=0, \ldots, n-2, \quad \text { where } \bar{B}_{k, i}^{\star}:=\sum_{r=i}^{n-1} \bar{B}_{k, i} \text { for } i=0, \ldots, n-1 .
$$


Expand the restrictions $Q_{\ell}^{k}$ (resp. $\bar{Q}_{\ell}^{k}$ ) of the B-spline-like $Q_{\ell}, \ell \in \mathbb{Z}$, (resp. $\bar{Q}_{\ell}, \ell \in$ $\left.\Lambda_{n-2}(\mathbb{K})\right)$ as follows

$$
Q_{\ell}^{k}=\sum_{i=0}^{n-1} \gamma_{\ell, k, i} V_{k, i}, \quad \bar{Q}_{\ell}^{k}=\sum_{i=0}^{n-2} \bar{\gamma}_{\ell, k, i} \bar{V}_{k, i} .
$$

Now, from (23) we know that

$$
\bar{Q}_{\ell}^{k}=D\left(\sum_{p \geqslant \ell} \bar{N}_{p}^{k}\right)
$$

Taking account of (29) and (27), we know that, for each $i=0, \ldots, n-2$, the coefficients $\bar{\gamma}_{\ell, k, i}$ can be deduced from the Bézier points of the functions $\sum_{p \geqslant \ell} \bar{N}_{p}^{k} \in L_{1}^{k} \mathbb{E}_{k}$ (see formula (5)). Combining (22) with the left part of (28) and with (26), we can write:

$$
\begin{aligned}
\sum_{p \geqslant \ell} \bar{N}_{p}^{k} & =\frac{1}{w_{1}^{k}} \sum_{p \geqslant \ell} Q_{p}^{k}=\frac{1}{w_{1}^{k}} \sum_{p \geqslant \ell}\left(\sum_{i=0}^{n-1} \gamma_{p, k, i} V_{k, i}\right)= \\
& =\frac{1}{w_{1}^{k}} \sum_{i=0}^{n-1}\left(\sum_{p \geqslant \ell} \gamma_{p, k, i}\right)\left(\frac{w_{1}^{k} \bar{B}_{k, i}}{\delta_{k, i}}\right)=\sum_{i=0}^{n-1}\left(\frac{\sum_{p \geqslant \ell} \gamma_{p, k, i}}{\delta_{k, i}}\right) \bar{B}_{k, i}
\end{aligned}
$$

It follows that

$$
\bar{\gamma}_{\ell, k, i}=\frac{\sum_{p \geqslant \ell} \gamma_{p, k, i+1}}{\delta_{k, i+1}}-\frac{\sum_{p \geqslant \ell} \gamma_{p, k, i}}{\delta_{k, i}}, \quad i=0, \ldots, n-2,
$$

that is, on account of (25) and of the left part of (28),

$$
\bar{\gamma}_{\ell, k, i}=\frac{\sum_{p \geqslant \ell} \gamma_{p, k, i+1}}{\sum_{p} \gamma_{p, k, i+1}}-\frac{\sum_{p \geqslant \ell} \gamma_{p, k, i}}{\sum_{p} \gamma_{p, k, i}}, \quad i=0, \ldots, n-2 .
$$

\subsubsection{One example}

To make the previous dimension diminishing procedure more tangible, we illustrate it with cardinal symmetric geometrically continuous quartic B-splines, see [33]. In other words, we take $t_{k}=k$ for all $k$, and each spline $S \in \mathbb{S}$ is continuous on $\mathbb{R}$, polynomial of degree at most four on each $\left[t_{k}, t_{k+1}\right]$, and it satisfies connection conditions of the form

$$
\left(S^{\prime}\left(t_{k}^{+}\right), S^{\prime \prime}\left(t_{k}^{+}\right), S^{\prime \prime \prime}\left(t_{k}^{+}\right)\right)^{T}=\left[\begin{array}{lll}
1 & 0 & 0 \\
b & 1 & 0 \\
\frac{b c}{2} & c & 1
\end{array}\right]\left(S^{\prime}\left(t_{k}^{-}\right), S^{\prime \prime}\left(t_{k}^{-}\right), S^{\prime \prime \prime}\left(t_{k}^{-}\right)\right)^{T}, \quad k \in \mathbb{Z},
$$

where $b, c$ are any real parameters. In [33], this spline space $\mathbb{S}$ was proved to be good for design if and only if the two conditions below are simultaneously satisfied:

$$
b+6>0, \quad c+4>0 .
$$


14

Carolina Vittoria Beccari et al.
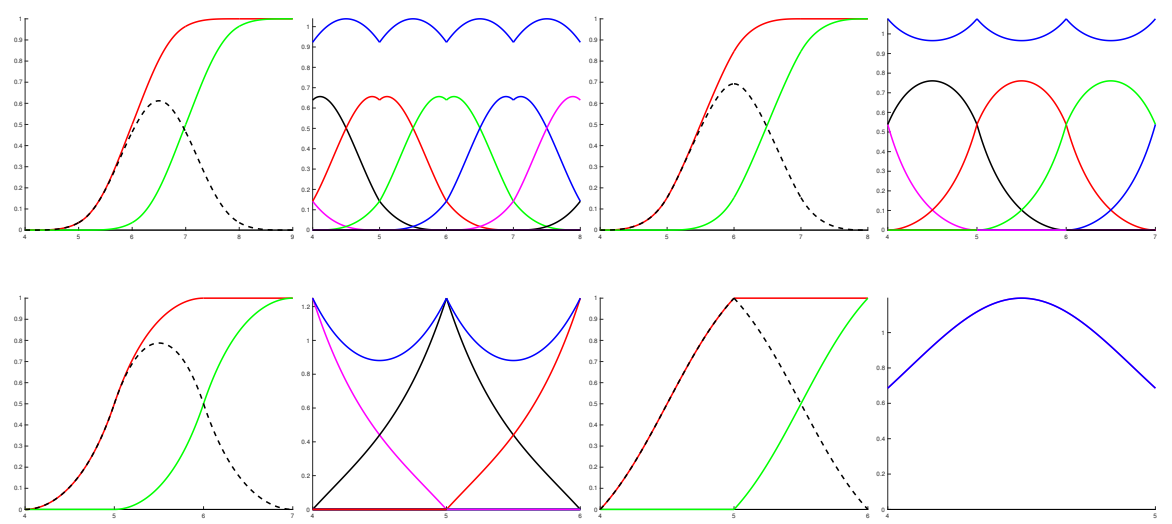

Fig. 1 Four steps of dimension diminishing in the quartic spline space of Subsection 2.3.3, with $b=c=1$.
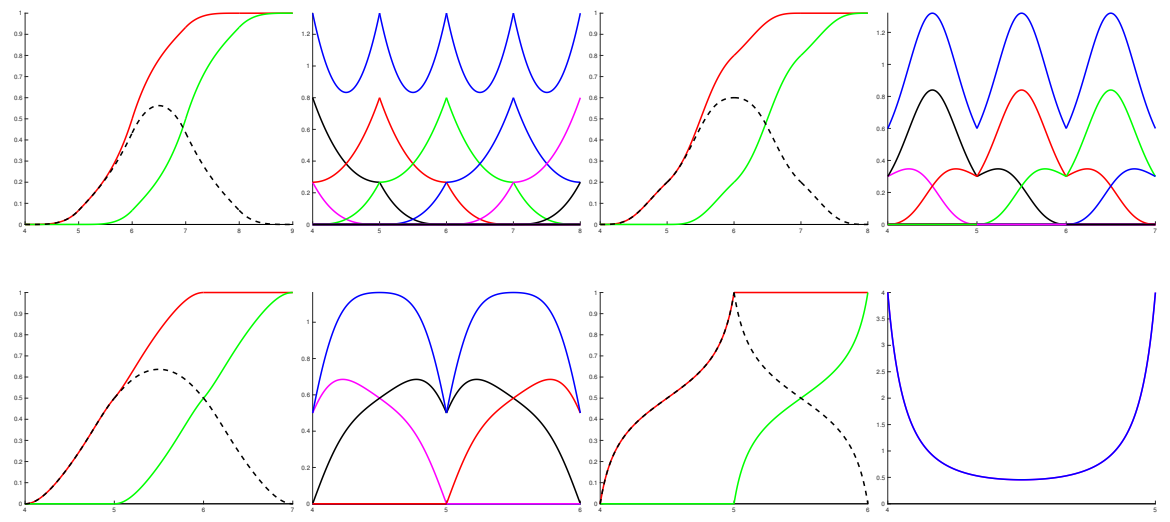

Fig. 2 Same as in Fig. 1, but with $b=-3, c=-2$.
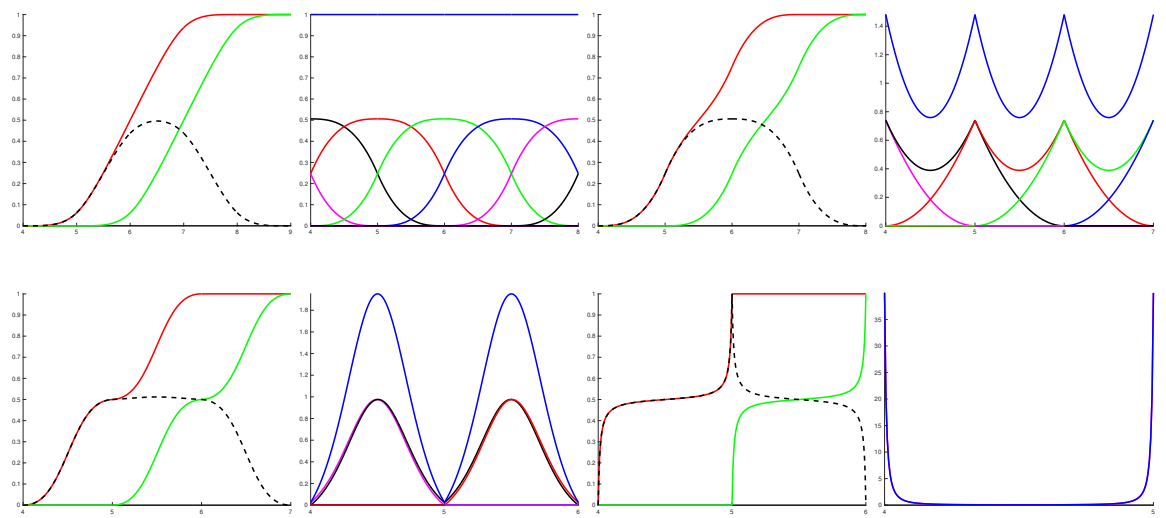

Fig. 3 Same as in Fig. 1, but with $b=0, c=-3.9$. 
Figs. 1, 2, and 3 contain eight pictures which two by two represent four steps of dimension diminishing, for various values of the parameters $b, c$ satisfying (33).

- The upper left pair of pictures concerns the initial spline space $\mathbb{S}$ and the PEC-spline space $D \mathbb{S}$. (respectively good for design and good for interpolation). On the left one, we can see one B-spline in $\mathbb{S}$, namely, $N_{4}$ with support $[4,9]$, along with the two consecutive functions $N_{4}^{\star}, N_{5}^{\star}$, to which it is linked via (12). The right one shows the B-spline-like basis $Q_{\ell}, \ell \in \mathbb{Z}$, in $D \mathbb{S}$, obtained from (13), along with the first positive piecewise function $w_{1}:=\sum_{\ell} Q_{\ell} \in D \mathbb{S}$ which produces the good for design PEC-spline space $L_{1} \mathbb{S}$ by piecewise division (here, geometrically continuous rational splines).

- Similar illustrations concerning $L_{1} \mathbb{S}$ and $D L_{1} \mathbb{S}$ are shown in the upper right pair of pictures, with in particular the B-spline of support $[4,8]$ in $L_{1} \mathbb{S}$, obtained by (22) and the positive piecewise function $w_{2} \in D L_{1} \mathbb{S}$ replacing $w_{1}$ and leading to the good for design PEC-spline space $L_{2} \mathbb{S}$ by piecewise division.

- The lower left pair concerns $L_{2} \mathbb{S}$ and $D L_{2} \mathbb{S}$, with the B-spline of support [4,7], and $w_{3} \in D L_{2} \mathbb{S}$ leading to the good for design PEC-spline space $L_{3} \mathbb{S}$ by piecewise division.

- Finally, the lower right pair concerns $L_{3} \mathbb{S}$ and $D L_{3} \mathbb{S}$, with the B-spline of support $[4,6]$, and $w_{4} \in D L_{3} \mathbb{S}$ leading to the good for design PEC-spline space $L_{4} \mathbb{S}$ by piecewise division. Note that $D L_{3} \mathbb{S}$ has one-dimensional section-spaces, and therefore on each interval $\left[t_{k}^{+}, t_{k+1}^{-}\right], w_{1}$ coincides with the only B-spline-like which is non-zero on that interval. As for $L_{4} \mathbb{S}$ it is a space of piecewise constant splines.

In the last picture of Fig. 3, we can see that, on the interval considered, the positive function $w_{4}$ is very close to zero. This is consistent with the fact that the value $c=$ -3.9 is close to the limit in (33).

\section{Is a given PEC-spline space good for design?}

In this section, we start with a given PEC-spline space $\mathbb{S}$, assuming that:

- for each $k \in \mathbb{Z}$, the section-space $\mathbb{E}_{k}$ is an $(n+1)$-dimensional EC-space good for design on $\left[t_{k}, t_{k+1}\right]$;

- the spline space $\mathbb{S}$ itself contains the constants.

Then, we want to build a numerical procedure to answer the question: is the PECspline space $\mathbb{S}$ good for design or not? The idea on which the test is based can be summarised as follows: if we can construct positive piecewise functions $w_{1} \in D \mathbb{S}$, then $w_{2} \in D L_{1} \mathbb{S}$ (where $L_{1}$ stands for the piecewise division by $w_{1}$ applied after piecewise differentiation), $w_{3} \in D L_{2} \mathbb{S}$, and so forth up to dimension one, while remaining within the class of PEC-spline spaces, then the initial space $\mathbb{S}$ will be good for design. Now, the present assumptions on $\mathbb{S}$ guarantee only that $D \mathbb{S}$ is a PEC-spline space. We do not know if we can find a positive $w_{1} \in D \mathbb{S}$. If ever such a spline $w_{1}$ exists, the corresponding spline space $L_{1} \mathbb{S}$ is a PEC-space containing the constants, but we cannot assert that $D L_{1} \mathbb{S}$ will be a PEC-spline space in turn, or equivalently we cannot assert that the section-spaces of $L_{1} \mathbb{S}$ will be good for design on their intervals. It is essential to take these difficulties into account in the construction of the test. 
3.1 Build a theoretical test

The theoretical test proposed below reinterprets the results recalled in the previous section. In particular, the observations above explain why, step by step, our algorithm mimics the procedure described in Subsection 2.3.2 and illustrated in Subsection 2.3.3. Furthermore, it will also be based on the necessary local positivity property (18). To facilitate the description, from now on, we assume here that $m_{k}=1$ for all $k \in \mathbb{Z}$. We will comment on this requirement later on.

\subsubsection{Step 0}

The initial part of the test concerns our initial spline space $\mathbb{S}$ along with the PECspline space $D \mathbb{S}$. It comprises two parts: the first one is connected to the possible existence of a B-spline basis in $\mathbb{S}$, while the second one tests the existence of a Bspline-like basis in $D \mathbb{S}$.

- Step 0.1: Towards a B-spline basis in $\mathbb{S}$. The first question to be answered is: can we find a normalised sequence $N_{\ell}, \ell \in \mathbb{Z}$, satisfying the support and endpoint properties? To avoid the question of normalisation we replace it by the following question, for each $\ell \in \mathbb{Z}$ : in the $2 n$-dimensional spline space $\mathbb{S}_{\ell, \ell+n}$, can we find a unique spline $N_{\ell}^{\star}$ such that

$$
N_{\ell}^{\star} \text { vanishes } n \text { times at } t_{\ell} \text { and }\left(\mathbb{I}-N_{\ell}^{\star}\right) \text { vanishes } n \text { times at } t_{\ell+n} \text { ? }
$$

We search for $N_{\ell}^{\star}$ by searching for the coefficients $\alpha_{\ell, k, i}^{\star}, k=\ell, \ldots, \ell+n-1, i=$ $0, \ldots, n$ such that

$$
N_{\ell \mid\left[t_{k}, t_{k+1}\right]}^{\star}=\sum_{i=0}^{n} \alpha_{\ell, k, i}^{\star} B_{k, i}, \quad \ell \leqslant k \leqslant \ell+n-1,
$$

with

$$
\alpha_{\ell, k, i}^{\star}= \begin{cases}0 & \text { for } k=\ell \text { and } i=0, \ldots, n-1, \\ 1 & \text { for } k=\ell+n-1 \text { and } i=1, \ldots, n .\end{cases}
$$

We then simply have to ensure the connection conditions at the interior knots $t_{k}, k=$ $\ell+1, \ldots, \ell+n-1$. Accordingly, answering question (34) consists in solving a linear system of $n(n-1)$ equations in $n(n-1)$ unknowns. We thus test the regularity of a square matrix of order $n(n-1)$ whose non-zero entries are the values of successive derivatives of the concerned Bernstein basis at the endpoints of their intervals.

If one among all the questions (34) obtains a negative answer, then the PEC-spline space $\mathbb{S}$ is not good for design, for it cannot possess a B-spline basis. Suppose that all answers are positive. Then, we proceed to step 0.2.

- Step 0.2: Possible existence of a B-spline-like basis in $D \mathbb{S}$. For each $\ell \in \mathbb{Z}$, we first extend each function $N_{\ell}^{\star}$ by 0 on the left of $t_{\ell}$ (with therefore all corresponding coefficients $\alpha_{\ell, k, i}^{\star}$ in (35) equal to 0) and by 1 on the right of $t_{\ell+n}$ (with therefore all corresponding coefficients $\alpha_{\ell, k, i}^{\star}$ in (35) equal to 1), while keeping the same notation. We now have at our disposal a bi-infinite sequence $N_{\ell}^{\star}, \ell \in \mathbb{Z}$, in $\mathbb{S}$, from which we can build the two sequences

$$
N_{\ell}:=N_{\ell}^{\star}-N_{\ell+1}^{\star} \in \mathbb{S}, \quad Q_{\ell}=D N_{\ell}^{\star} \in D \mathbb{S}, \quad \ell \in \mathbb{Z} .
$$


The left sequence is the only possible candidate to be the B-spline basis of $\mathbb{S}$, it is normalised and satisfies the support property, along with the endpoint property, the exactness of the number of vanishing conditions being omitted.

Consider the right sequence in (36). As in the previous section, we use the notation $Q_{\ell}^{k}$ for the restriction of the piecewise function $Q_{\ell}$ to $\left[t_{k}^{+}, t_{k+1}^{-}\right]$. Given any $\ell, k \in \mathbb{Z}$, we expand the function $Q_{\ell}^{k}$ in the Bernstein-like basis $\left(V_{k, 0}, \ldots, V_{k, n-1}\right)$ in $D \mathbb{E}_{k}$ as in (28). Then, according to (36), (35), and (5), we know that

$$
\gamma_{\ell, k, i}=\alpha_{\ell, k, i+1}^{\star}-\alpha_{\ell, k, i}^{\star}, \quad i=0, \ldots, n-1, \quad \ell \in \mathbb{Z} .
$$

The question we have to answer is

Do all coefficients $\gamma_{\ell, k, i}$ satisfy the positivity property (18)?

If the answer to (38) is negative, then the test stops: the PEC-spline space $\mathbb{S}$ is not good for design. If the question (38) obtains a positive answer, then we can state that the sequence $Q_{\ell}, \ell \in \mathbb{Z}$, is indeed a B-spline-like basis in the PEC-spline space $D \mathbb{S}$.

Suppose that all questions of Step 0 have received a positive answer. Then we proceed to Step 1 .

\subsubsection{Step 1}

Consider the spline $w_{1} \in D \mathbb{S}$ defined according to (21). For each $k \in \mathbb{Z}$, its restriction $w_{1}^{k}$ to $\left[t_{k}^{+}, t_{k+1}^{-}\right]$can be expanded as

$$
w_{1}^{k}=\sum_{i=0}^{n-1} \delta_{k, i} V_{k, i}, \quad \text { with } \delta_{k, i}:=\sum_{\ell} \gamma_{\ell, k, i}>0 \text { for } i=0, \ldots, n-1,
$$

the positivity indicated in (39) resulting from the positive answer to (38). Then, denoting by $L_{1}^{k}$ the differentiation followed by division by $w_{1}^{k}$, from Theorem 3 we can assert that $L_{1}^{k} \mathbb{E}_{k}$ is an $n$-dimensional EC-space good for design on $\left[t_{k}, t_{k+1}\right]$. Equivalently, we can assert that the spline $w_{1} \in D \mathbb{S}$ is piecewise positive on $(\mathrm{I} ; \mathbb{T})$, and that the PEC-spline space $L_{1} \mathbb{S}$ obtained by piecewise division of $D \mathbb{S}$ by $w_{1}$ satisfies the same properties as $\mathbb{S}$ with $n$ replaced by $(n-1)$ (and each $\mathbb{E}_{k}$ replaced by $L_{1}^{k} \mathbb{E}_{k}$ ). We can thus apply Step 0 to the PEC-spline space $L_{1} \mathbb{S}$. This is Step 1 of the test which thus concerns $L_{1} \mathbb{S}$ and $D L_{1} \mathbb{S}$. Nevertheless, the following observations reduces it to very little.

- Step 1.1: It should be observed that this part of Step 1 is useless. Indeed, in $L_{1} \mathbb{S}$, we can consider the two bi-infinite sequences of splines

$$
\bar{N}_{\ell}:=\frac{Q_{\ell}}{w_{1}}, \quad \bar{N}_{\ell}^{\star}:=\sum_{p \geqslant \ell} \bar{N}_{p} \in L_{1} \mathbb{S}, \quad \ell \in \mathbb{Z} .
$$

The splines $\bar{N}_{\ell}, \ell \in \mathbb{Z}$, form the B-spline basis of $L_{1} \mathbb{S}$ and their local expansions satisfy the positivity property (18) as does the B-spline-like basis $Q_{\ell}, \ell \in \mathbb{Z}$.

- Step 1.2: The next part of Step 1 concerns the splines

$$
\bar{Q}_{\ell}:=D \bar{N}_{\ell}^{\star} \in D L_{1} \mathbb{S}, \quad \ell \in \mathbb{Z} .
$$


For each $k \in \mathbb{Z}$, we can successively introduce the Bernstein basis $\left(\bar{B}_{k, 0}, \ldots, \bar{B}_{k, n-1}\right)$ in $L_{1}^{k} \mathbb{E}_{k}$, and the Bernstein-like basis $\left(\bar{V}_{k, 0}, \ldots, \bar{V}_{k, n-2}\right)$ in $D L_{1}^{k} \mathbb{E}_{k}$, exactly as we did in Subsection 2.3.2. We then have to check that all local expansions

$$
\bar{Q}_{\ell}=\sum_{i=0}^{n-2} \bar{\gamma}_{\ell, k, i} \bar{V}_{k, i}
$$

satisfy the expected positivity property. Here, it reduces to the local expansions on all intervals $\left[t_{k}, t_{k+1}\right]$ contained in $] t_{\ell}, t_{\ell+n-1}[$, that is, $k=\ell+1, \ldots, \ell+n-2$. Note that the corresponding coefficients $\bar{\gamma}_{\ell, k, i}$ are obtained from the coefficients $\gamma_{\ell, k, i}$ computed at Step 0.1 via formula (31). If at least one of them is not positive, the test stops: $\mathbb{S}$ is not good for design. Otherwise we continue.

\subsubsection{Up to Step $n-1$ :}

If the test reaches Step 1, that is, if the coefficients

$$
\gamma_{\ell, k, i}^{\{0\}}:=\gamma_{\ell, k, i}
$$

satisfy the local expansion positivity property (18), then the additional possible steps can be summarised as follows: if, for some $p \geqslant 1$, we have obtained coefficients $\gamma_{\ell, k, i}^{\{p-1\}}$ which satisfy the local expansion positivity property (18), then, compute the coefficients

$$
\gamma_{\ell, k, i}^{\{p\}}=\frac{\sum_{p \geqslant \ell} \gamma_{p, k, i+1}^{\{p-1\}}}{\sum_{p} \gamma_{p, k, i+1}^{\{p-1\}}}-\frac{\sum_{p \geqslant \ell} \gamma_{p, k, i}^{\{p-1\}}}{\sum_{p} \gamma_{p, k, i}^{\{p-1\}}}, \quad i=0, \ldots, n-p, \quad \ell, k \in \mathbb{Z},
$$

and answer the question

$$
\text { Do all coefficients } \gamma_{\ell, k, i}^{\{p\}} \text { satisfy the positivity property (18)? }
$$

If at some level $p \leqslant n-2$, we obtain at least one negative answer, then the step stops at level $p$. Suppose that we can successfully go through all the previous iterations up to Step $(n-2)$. We have thus obtained a PEC-spline space $L_{n-1} \mathbb{S}$ which contains constants. Here, because we are working with simple knots, $L_{n-1} \mathbb{S}$ is automatically good for design. The spline space $\mathbb{S}$, obtained from $L_{n-1} \mathbb{S}$ by alternating multiplications by positive piecewise functions and integrations, is thus good for design.

If the test is successful, afterwards we can say that the sequence $N_{\ell}, \ell \in \mathbb{Z}$, determined at Step 0, see (36), is the B-spline basis of $\mathbb{S}$.

\subsection{The numerical test}

The numerical test follows in a precise way the description of the various steps in the previous subsection. Of course, the passage from one step to the next one takes numerical aspects into account as follows. 
1. In Step 0.1, if any of the matrices involved is nearly-singular or/and very illconditioned, we stop the test: in that case the corresponding system is not solvable or the solution of (34) cannot be computed with sufficient accuracy.

2. Similarly, all positivity tests mentioned earlier are actually replaced by $\gamma_{\ell, k, i}^{\{p\}}>$ tol, where tol is a small positive number (in our experiments, tol $=1 e-30$ ).

When we stop at some level $p \leqslant n-2$, then, numerically speaking, we consider that the PEC-spline space $\mathbb{S}$ is not good for design. When the test successfully reaches $p=n-1$, we conclude that $\mathbb{S}$ is good for design, numerically speaking.

Subsequently, we both illustrate the test in itself and comment on it.

- Suppose that the numerical test has been successfully applied to a given PEC-spline space $\mathbb{S}$. Then, not only do we know that $\mathbb{S}$ is good for design, but we even have determined its B-spline basis $N_{\ell}, \ell \in \mathbb{Z}$, at Step 0 of the test. Therefore, this enables us to easily obtain all parametric curves in $\mathbb{R}^{d}$ as

$$
S(x)=\sum_{\ell} N_{\ell}(x) P_{\ell}, \quad x \in \mathbb{R},
$$

where the $P_{\ell}$ 's are any given points in $\mathbb{R}^{d}, d \geqslant 1$. Obviously, the knowledge of the $\mathrm{B}$-spline bases can also be exploited in many other problems if needed, e.g., interpolation problems.

- The test was first used to rediscover the conditions for a given PEC-spline space $\mathbb{S}$ to be good for design in many situations for which explicit necessary and sufficient conditions (concerning the parameters on which $\mathbb{S}$ depends) had previously been obtained, with $n=4$, for instance in [33]. In the many examples investigated, we could observe that the test numerically confirms the conditions with a remarkable precision. - We now would like to illustrate the various steps where the test can stop. This is done with $n=4$. In the present comments, the space $\mathbb{S}$ is composed of $C^{3}$ splines, with regularly spaced knots, and knot spacing $h=t_{k+1}-t_{k}$. For each $k$, the sectionspace $\mathbb{E}_{k}$ is the restriction to $\left[t_{k}, t_{k+1}\right]$ of the kernel $\mathbb{E}=\operatorname{ker} L$ of a linear differential operator $L$ of order five, with constant coefficients and odd characteristic polynomial $p_{L}$ having at least one non-real root. In that case, as an application of Theorem 1, it was proved in [8] that the spline space is good for design if and only if

$h<\frac{\pi}{M}, \quad$ where $M$ is the maximum of the positive imaginary parts of the roots of $p$.

We retrieve these conditions through the test. Before applying the test, we first have to know the so-called critical length for design of the space $\mathbb{E}$, that is, the positive number $\ell_{L}<+\infty$ such that $\mathbb{E}$ is an EC-space good for design if and only if $h<\ell_{L}$ $[10,30]$. In the first example, $L=D^{5}+a^{2} D^{3}$, with $a>0$. Hence, $\mathbb{E}$ is spanned by the five functions $1, x, x^{2}, \cos (a x), \sin (a x)$, and $\mathbb{S}$ depends on the two parameters $a, h$. It is well-known that $\ell_{L}=\frac{2 \pi}{a}[10]$, and the corresponding limit hyperbola $h<\frac{2 \pi}{a}$ is indicated in red in the plane $(a ; h)$ in Fig. 4, while the good for design condition (42) for $\mathbb{S}$ corresponds to the green region limited by the hyperbola branch $h<\frac{\pi}{a}$. In the second example, $L=D^{5}+\left(a^{2}+1\right) D^{3}+a^{2} D$, and $\mathbb{E}$ is spanned by $1, \cos x, \sin x, \cos (a x), \sin (a x)$, with $a>1$. The red curve, composed of two different 

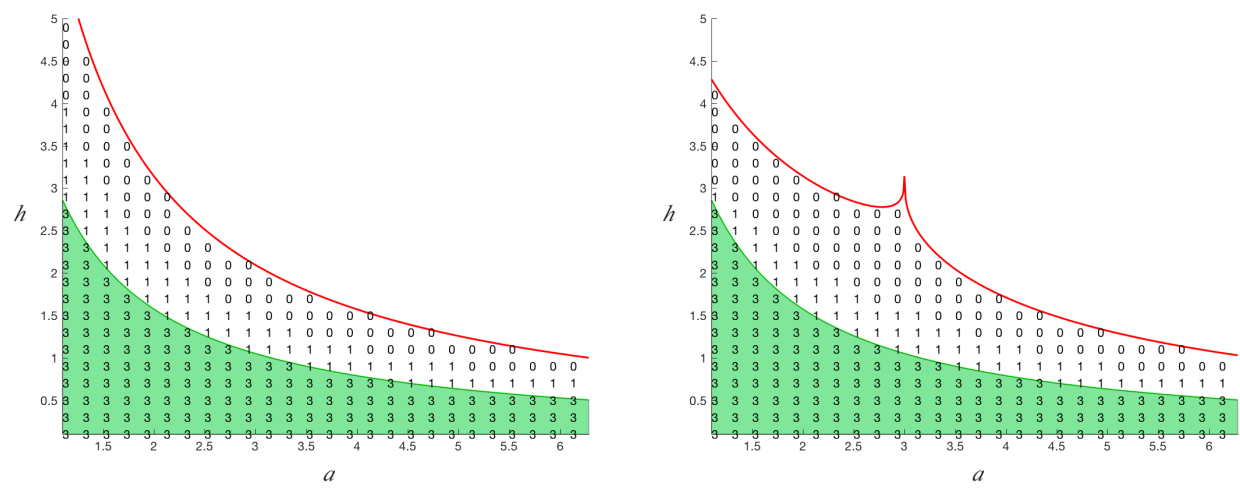

Fig. 4 The results of the test for $C^{3}$ splines with all sections taken from the space spanned by the five functions $1, x, x^{2}, \cos (a x), \sin (a x)$ (left) and $1, \cos x, \sin x, \cos (a x), \sin (a x)$ (right), depending on the pair $(a ; h)$

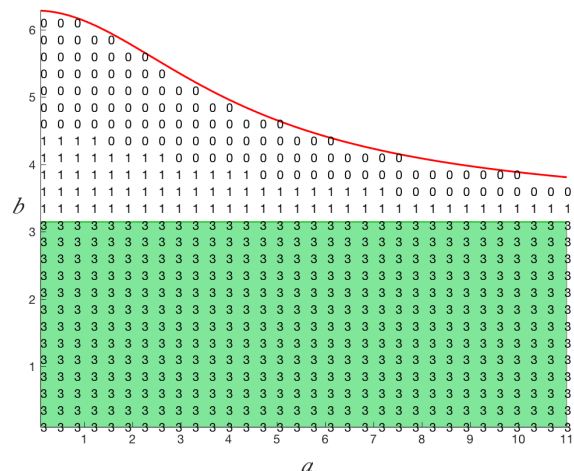

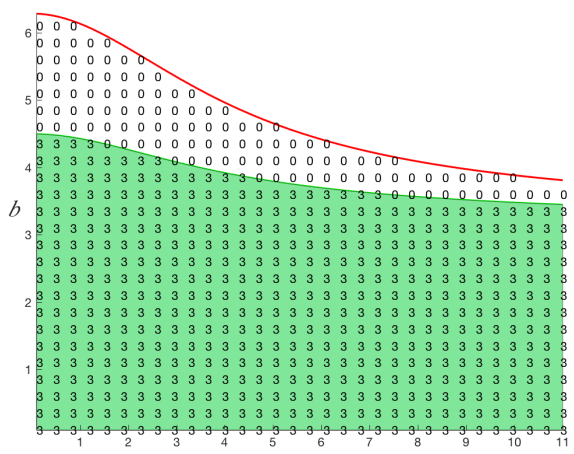

a

Fig. 5 The results of the test for $C^{3}$ splines with knot spacing $h=1$ and all sections taken from the space spanned by the five functions $1, \cosh (a x), \sinh (a x), \cos (b x), \sin (b x)$, depending on the pair $(a ; b)$. Left: simple knots. Right: alternatively simple and double knots.

parts meeting at $a=3$, corresponds to the starting condition $h<\ell_{L}$ (see [7]). In both examples, the number $m$ appearing in Fig. 4 at a given pair $(a ; h)$ indicates that, for these values of the two parameters, the test stops at Step $m$. In both examples, with the sampling used, we can see a number of pairs of parameters with "1". That the test successfully passed Step 0 implies that the spline $L_{1} \mathbb{S}$ does possess a B-spline basis. Still it is not good for design (otherwise $\mathbb{S}$ itself would be good for design). The test definitely shows that the presence of a B-spline basis in a PEC-spline space $\mathbb{S}$ does not guarantee that each spline space obtained from $\mathbb{S}$ by insertion of knots will possess a B-spline basis in turn, thus giving a negative answer to a frequently asked question.

- We have presented the test with simple knots only, in order to never meet discontinuities during the dimension diminishing process, and therefore at all stages we were working within the condition $\Lambda_{p}(\mathbb{K})=\mathbb{Z}$. It is nevertheless valid with any multi- 
plicities. If, at some step $r$, the spline space $L_{r} \mathbb{S}$ presents discontinuities, we simply split $L_{r} \mathbb{S}$ into spaces of the form $L_{r} \mathbb{S}_{k}^{-}, L_{r} \mathbb{S}_{k}^{+}$, or $L_{r} \mathbb{S}_{k, k^{\prime}}$, with $k<k^{\prime}$, with all interior multiplicities bounded above by $(n-r)$ and continue separately in each of these PEC-spline spaces. In Fig. 5 we illustrate this with knot spacing $h=1$, and with $L:=D^{5}+\left(b^{2}-a^{2}\right) D^{3}-a^{2} b^{2} D, a, b>0$, implying that $\mathbb{E}=\operatorname{ker} L$ is spanned over $\mathbb{R}$ by the five functions $1, \cosh (a x), \sinh (a x), \cos (b x), \sin (b x)$. In the left picture, all knots are simple, while in the right one we alternate simple and double knots. In both pictures the pairs $(a ; b)$ located below the red curve are those which ensure $1<\ell_{L}$ : from [7] we know that they satisfy either $b \leqslant \pi$, or

$$
\pi<b<2 \pi \quad \text { and } \quad\left(b^{2}-a^{2}\right) \sinh a \sin b+2 a b(\cosh a \cos b-1)<0 .
$$

In both pictures the good for design region is indicated in green. In the left case, it is described by $b<\pi$, in accordance with (42). In the right one, it is formed by all pairs $(a ; b)$ such that either $b \leqslant \pi$, or

$$
\pi<b<x_{0} \text { and } \quad \frac{1}{a} \tanh a>\frac{1}{b} \tan b
$$

where $\left.x_{0} \in\right] \pi, \frac{3 \pi}{2}\left[\right.$ satisfies $\tan x_{0}=x_{0}$. This confirms the results described in [8], see also [7].

- The test can be applied as well to PEC-splines on closed bounded intervals, or equivalently, to any restriction $\mathbb{S}_{k, k^{\prime}}$, with $k^{\prime}>k+1$, and $m_{k}=m_{k^{\prime}}=n+1$. If we additionally assume that $m_{j}=0$ for $k<j<k^{\prime}$, then the PEC-spline space $\mathbb{S}_{k, k^{\prime}}$ is $(n+1)$-dimensional. In this case, testing if $\mathbb{S}_{k, k^{\prime}}$ is good for design amounts to testing if the $n$-dimensional space $D \mathbb{S}_{k, k^{\prime}}$ is an Extended Chebyshev Piecewise Space (for short, ECP-space) on $\left(\left[t_{k}, t_{k^{\prime}}\right] ;\left(t_{k+1}, \ldots, t_{k^{\prime}-1}\right)\right.$ ) (same bound on the number of zeroes as in each section-space), or as well, if the piecewise space $\mathbb{S}_{k, k^{\prime}}$ is an ECP-space good for design on $\left(\left[t_{k}, t_{k^{\prime}}\right] ;\left(t_{k+1}, \ldots, t_{k^{\prime}-1}\right)\right)$. This coincides with the test developed in [2].

\section{Applications}

In this section we present a few results obtained via the numerical test which efficiently illustrate its interest.

\subsection{Shape effects with $C^{n-1}$ polynomial splines of degree $n$}

Polynomial splines defined by a certain degree and a given knot-vector $\mathbb{K}$ do not provide any shape parameters: a given control polygon produces one unique parametric spline curve. This inconvenience can be overcome by the insertion of knots of multiplicity zero, and of associated connection matrices (different from the identity). The knot-vector $\mathbb{K}$ remains unchanged, and therefore the number of poles too. A given control polygon now produces infinitely many different spline curves, the entries of the inserted connection matrices serving as shape parameters. 


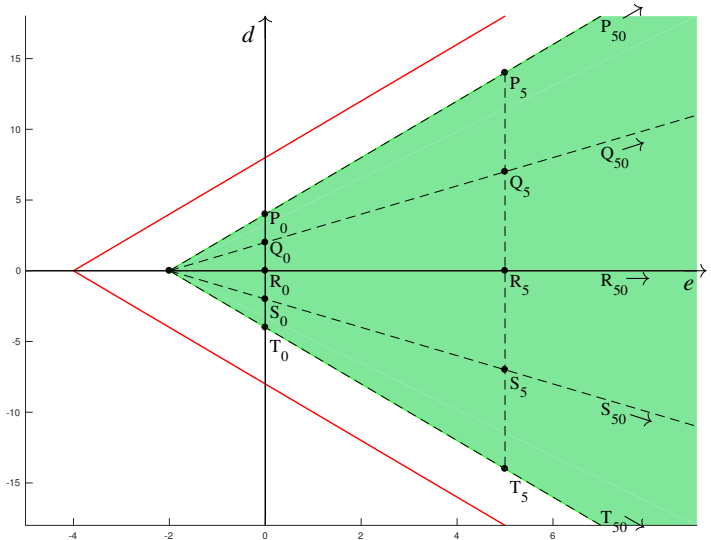

Fig. 6 Good for design region for $C^{2}$ cubic splines with knot spacing $1, m_{2 k}=1, m_{2 k+1}=0$ for all $k$, and at each knot $t_{2 k+1}$ the connection matrices (44) (above the symmetry line of the control polygon, see Fig. 7) and (45) (below the symmetry line).
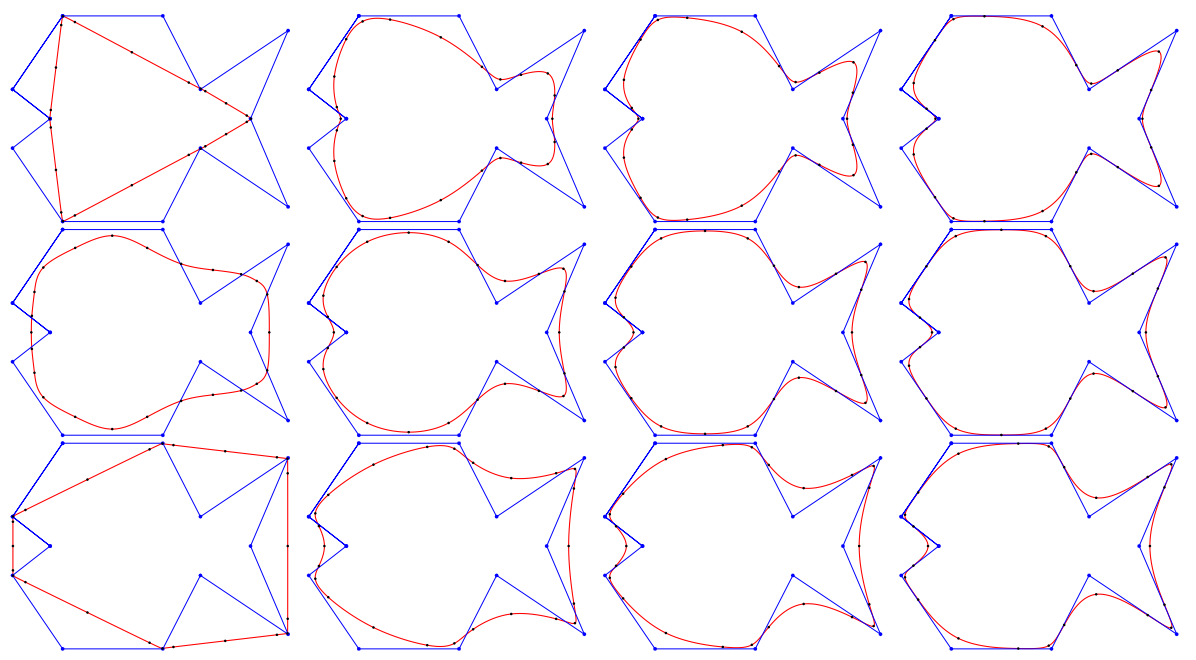

Fig. $7 C^{2}$ cubic splines with knot spacing $1, m_{2 k}=1, m_{2 k+1}=0$ for all $k$, and at each knot $t_{2 k+1}$ connection condition (44) (above symmetry line) and (45) (below symmetry line). From left to right: $e=-1.99 ; 0 ; 5 ; 50$. Top left: $d=-2(e+2)+\varepsilon$, with $\varepsilon=5 \times 10^{-5}$. Bottom left: $d=2(e+2)-\varepsilon$. Elsewhere, $d=\alpha(e+2)$, with $\alpha=-1$ (first row), $\alpha=0$ (second row), and $\alpha=1$ (third row). See Fig. 6 and comments in the text.

Introducing knots of zero multiplicities has already proved to be very useful, see e.g., [16]. Here, with the help of the numerical test, we illustrate this with $C^{2}$ cubic splines alternating simple knots with knots of multiplicity 0 . We assume that the knots are regularly spaced, with knot spacing equal to one, and with

$$
m_{2 k}=1, \quad m_{2 k+1}=0, \quad k \in \mathbb{Z} .
$$

The knot-vector $\mathbb{K}=\left(\xi_{\ell}\right)_{\ell \in \mathbb{Z}}$ is obtained with $\xi_{\ell}:=t_{2 \ell}=2 \ell$ for all $\ell$. In this situation, a pole $P_{k}$ represents the knot $t_{2 k+2}$ which is the middle of the support of the B-spline 
$N_{k}$. One can therefore represent the knot $t_{2 k+1}$ by the segment $P_{k-1} P_{k}$. Our control polygon (see Fig. 7) presenting an horizontal symmetry line, in order to preserve symmetry and the $C^{2}$ connection, at a knot of odd index the connection condition is

$$
\left(S^{\prime}\left(t_{2 k+1}^{+}\right), S^{\prime \prime}\left(t_{2 k+1}^{+}\right), S^{\prime \prime \prime}\left(t_{2 k+1}^{+}\right)\right)^{T}=M_{2 k+1}\left(S^{\prime}\left(t_{2 k+1}^{-}\right), S^{\prime \prime}\left(t_{2 k+1}^{-}\right), S^{\prime \prime \prime}\left(t_{2 k+1}^{-}\right)\right)^{T}
$$

with, on each segment above the symmetry line,

$$
M_{2 k+1}:=\left[\begin{array}{lll}
1 & 0 & 0 \\
0 & 1 & 0 \\
d & e & 1
\end{array}\right]
$$

and on each segment below the symmetry line,

$$
M_{2 k+1}:=\left[\begin{array}{ccc}
1 & 0 & 0 \\
0 & 1 & 0 \\
-d & e & 1
\end{array}\right]
$$

For the corresponding spline space $\mathbb{S}$ to be good for design it is necessary that the four-dimensional space obtained by restriction of $\mathbb{S}$ to $\left[t_{2 k}, t_{2 k+2}\right]$ be an ECP-space good for design on $\left(\left[t_{2 k}, t_{2 k+2}\right] ; t_{2 k+1}\right)$. We therefore first apply the ECP test developed in [2] to such two consecutive sections. We obtain that, numerically, the corresponding good for design region is characterised by

$$
|d|<2(e+4) .
$$

In the plane $(e ; d)$, this corresponds to the angular sector delimited by the red boundary and containing the origin, see Fig. 6. To simplify our work, we apply the numerical test only within this region, and we can state the following numerical characterisation.

Proposition 2 (proved numerically) The space of $C^{2}$ cubic splines $\mathbb{S}$ defined above is good for design if and only if

$$
|d|<2(e+2) \text {. }
$$

Within the good for design region (46), represented in green in Fig. 6, the two parameters $d, e$ act as shape parameters. The effects they produce are illustrated in Fig. 7, where we can see the variations in shape along several vertical lines. From top to bottom, the leftmost column represents three points of the line $e=-1.99$ in the good for design region (not indicated in Fig. 6 for the sake of clarity): the point $P_{-1.99}$ on the upper boundary, the point $R_{-1.99}$ on the horizontal axis, and finally the point $T_{-1.99}$ on the lower boundary (of course, we have to slightly move $P_{-1.99}$ and $T_{-1.99}$ to be inside the green region). Along the two boundary lines, the curves visually remain unchanged. In other words, adding both the top leftmost curve above each of the remaining columns and the bottom leftmost curve below it, we obtain, from top to bottom, the curves at the five points $P_{e}, Q_{e}, R_{e}, S_{e}$, and $T_{e}$ along the given vertical line of abscissa $e$ with, from left to right, $e=0, e=5, e=50$, see Fig. 6 . Note that, for $d=0$ (middle line in Fig. 6) we obtain a cardinal symmetric B-spline basis. 
There is no difficulty in generalising the process to $C^{n-1}$ splines of degree $n$, the connection matrix having entries at each knot of mutiplicity zero differing from the identity matrix only by its non-diagonal entries on the last row. It is also possible to insert zero multiplicities only at certain places in order to obtain local rather than global shape effects.

\subsection{Design versus interpolation with symmetric cardinal polynomial B-splines}

In this subsection, we would like to illustrate the fact that the test can also be used for interpolation purposes, via the crucial link between interpolation and design established in [32] and reminded in Theorem 5. With simple knots $t_{k}=k$ for all $k \in \mathbb{Z}$, we consider a space $\widehat{\mathbb{S}}$ of cardinal geometrically continuous quintic splines preserving symmetry. We know that $\widehat{\mathbb{S}}$ is good for interpolation if and only if the space $\widehat{\widehat{S}}$ of degree six splines obtained by integration of $\widehat{\mathbb{S}}$ is good for design. In other words, to answer the question: can we use $\widehat{\mathbb{S}}$ to solve Hermite interpolation problems satisfying the Schoenberg-Whitney conditions?, we apply our numerical test to $\widehat{\mathbb{S}}$. Each $\widehat{S} \in \widehat{\mathbb{S}}$ satisfies connection conditions of the form, see [33]

$$
\left(\widehat{S}^{\prime}\left(t_{k}^{+}\right), \ldots, \widehat{S}^{(4)}\left(t_{k}^{+}\right)\right)^{T}=\left[\begin{array}{cccc}
1 & 0 & 0 & 0 \\
a & 1 & 0 & 0 \\
\frac{a b}{2} & b & 1 & 0 \\
d & \frac{b c}{2} & c & 1
\end{array}\right]\left(\widehat{S}^{\prime}\left(t_{k}^{-}\right), \ldots, \widehat{S}^{(4)}\left(t_{k}^{-}\right)\right)^{T}, \quad k \in \mathbb{Z}
$$

In the spline space $\widehat{\widehat{S}}$, at each knot the lower triangular connection matrix is obtained by addition of the column $(1,0, \ldots, 0)^{T}$ on the left of the matrix in (47). Since it is not reasonable to handle four parameters at the same time, from now on we assume that $a=d=0$, implying that $\widehat{\mathbb{S}}$ is composed of $C^{2}$ splines. This choice facilitates the numerical search for the good for design region in the plane $(b ; c)$. Indeed, we can start with the symmetric cardinal spline space $\mathbb{S}$ of cardinal geometrically continuous quartic splines already used in Subsection 2.3.3 to illustrate the dimension diminishing process within the condition "good for design". We recall that, for $\mathbb{S}$ to be good for design, it is necessary and sufficient that the two conditions in (33) hold true. The spline space $\widehat{\widehat{\mathbb{S}}}$ being obtained from $\mathbb{S}$ under two integration steps, we know $\widehat{\widehat{\mathbb{S}}}$ is good for design at least on the region of the plane $(b ; c)$ described by (33). We thus simply have to apply the test outside this region, and the many points tried clearly indicate that we cannot go beyond (33). Numerically speaking, the two conditions (33) are therefore necessary and sufficient for $\widehat{\widehat{\mathbb{S}}}$ to be good for design. Therefore we can state the following numerical characterisation (to be compared with Theorem 5).

Proposition 3 (proved numerically) For the cardinal quintic spline space $\widehat{\mathbb{S}}$ defined by (47) with $a=d=0$, the following three properties are equivalent:

1. $\widehat{\mathbb{S}}$ is good for interpolation;

2. $\widehat{\mathbb{S}}$ is good for design;

3. the parameters $b, c$ satisfy (33), that is, $b+6>0$ and $c+4>0$. 
A control polygon being given, we first illustrate design in $\widehat{\mathbb{S}}$ within conditions (33) in Fig. 8. We can summarise the shape effects obtained as follows:

- for a given value of $b \in]-6,+\infty[$, increasing the value of $c$ significantly improves the curve, in the sense that it better imitates the control polygon;

- from this point of view, the effect of increasing $c$ is all the stronger as $b$ is close to its boundary $b=-6$.

The most remarkable point to retain from this example is the fact that the obtained curves mimick the control polygon all the better as the parameter $b$ gets closer to its lower bound -6 . This gives further evidence of the interest of not limiting the connection matrices to be totally positive (see [21]), contrary to what was initially done for geometrically continuous polynomial splines [13,12], and also [1,34].

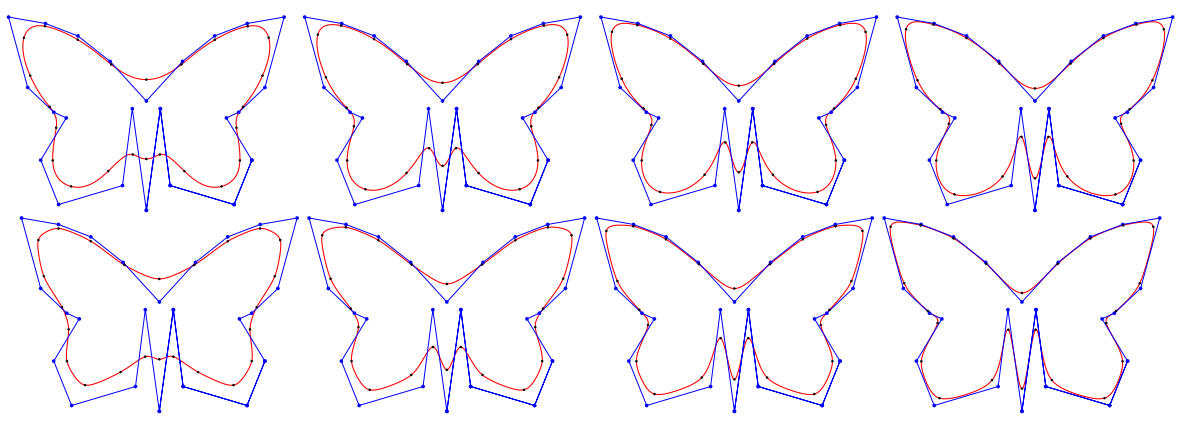

Fig. 8 Design with cardinal symmetric $C^{2}, F^{4}$ quintic B-splines with connections (47) and $a=d=0$ From left to right, $c=-3.99 ; 0 ; 10 ; 100$. Up: $b=0$ (i.e., $C^{3}, F^{4}$ splines, and even the ordinary $C^{4}$ splines for $c=0)$. Down: $b=-5.99$.

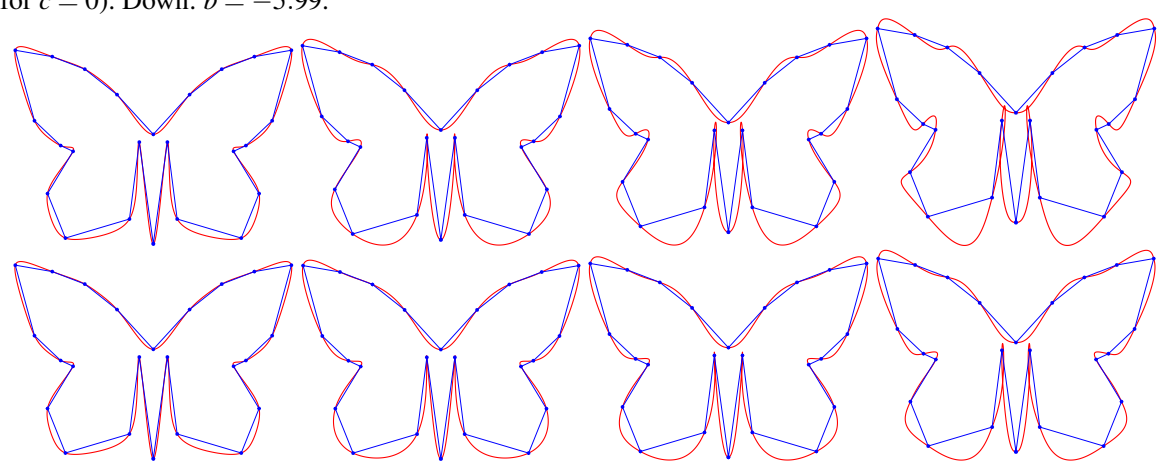

Fig. 9 Lagrange interpolation with symmetric cardinal $C^{2}, F^{4}$ polynomial quintic B-splines with, at each knot, with $a=d=0$ in the connection conditions (47). From left to right, $b=-5.99 ; 0$ (i.e., $C^{3}$ splines); 10; 100. Up: $c=0$ (ordinary $C^{4}$ quintic splines for $b=0$ ). Down: $c=100$.

Below we assume again that $b+6>0$ and $c+4>0$. Under Schoenberg-Whitney conditions, any Hermite interpolation problem in $\widehat{\mathbb{S}}$ has a unique solution. It should be observed that this result guarantees nothing about the quality of the obtained solution. Nevertheless, hopefully it might be possible to use the two parameters $b, c$ to improve the interpolating curves. This is illustrated with Lagrange interpolation at the knots in 
$\widehat{\mathbb{S}}$. The vertices of the previous control polygon now serve as the points to interpolate. Only a small selection of the many interpolating curves we tried is presented, and in particular the too oscillating ones are avoided. According to our experiments, we can summarise the effects of the two parameters as follows:

- along the boundary $b=-6$, there is no visual difference between all the curves when $c$ ranges over $]-4,+\infty[$ (see, e.g., the two leftmost curves);

- for a given value of $c \in]-4,+\infty[$, increasing the value of $b$ increases the presence/amplitude of oscillations, loops, self intersections, etc;

- oppositely, for a given value of $b$ not too close to $-6^{+}$, increasing the value of $c$ significantly reduces them.

In particular, we would like to draw the reader's attention to the presence of loops in the ordinary quintic spline curve $(b=c=0)$. Increasing $c$ makes them disappear and it also reduces the amplitude of the oscillations (see $b=0, c=100$ for comparison). As is usual, these observations are in accordance with the design analysis above. Again, we can say that the best (i.e., the least oscillatory) curves are obtained along the limit $b=-6^{+}$.

Remark 4 It should be observed that, numerically speaking, Proposition 3 is still valid when replacing the space $\widehat{\mathbb{S}}$ by the initial space $\mathbb{S}$, except that, in that case, the equivalence between the last two properties is an exact one, proved in [33], as reminded in Subsection 2.3.3. As a matter of fact, from a few more applications of the test in higher dimensions, we conjecture that Proposition 3 remains valid after any number of integration steps.

\subsection{L-splines in higher dimensions}

As mentioned in Subsection 3.2, $C^{3}$ splines with all pieces taken from the kernel $\mathbb{E}=\operatorname{ker} L$ of a linear differential operator of the form $L=D^{5}+a_{2} D^{3}+a_{0} D$, where $a_{0}, a_{2} \in \mathbb{R}$, were investigated in [8], see more generally [19]. If the characteristic polynomial has only real roots, the space $\mathbb{E}$ is an EC-space good for design on the whole of $\mathbb{R}$, and therefore under parametric continuity conditions, any associated spline space $\mathbb{S}$ is a Chebyshevian spline space, and is automatically good for design. This is the reason why we focussed on the case where not all roots of the characteristic polynomial are real. In that case, we are dealing with PEC-splines, at least provided that the difference between any two consecutive knots is less than the critical length for design. With simple equispaced knots, we know that (42) is the necessary and sufficient condition for $\mathbb{S}$ to be good for design. In [8] it was conjectured that, with simple equispaced knots, (42) could remain the necessary and sufficient condition in higher dimension too. Here, we consider a space $\mathbb{S}$ of $C^{5}$ splines with all pieces taken from $\mathbb{E}=\operatorname{ker} L$, with $L=D^{7}+a_{4} D^{5}+a_{2} D^{3}+a_{0} D$, where $a_{0}, a_{2}, a_{4} \in \mathbb{R}$. This class contains twenty-two different subclasses of spaces $\mathbb{E}$, among which only seven real cases for which $\mathbb{S}$ is a space of Chebyshevian splines (i.e., all roots of the characteristic polynomial are real).

Our aim was to use the test to either strengthen or contradict the conjecture. So far we have tested successfully a number of spaces in different non-real subclasses: 


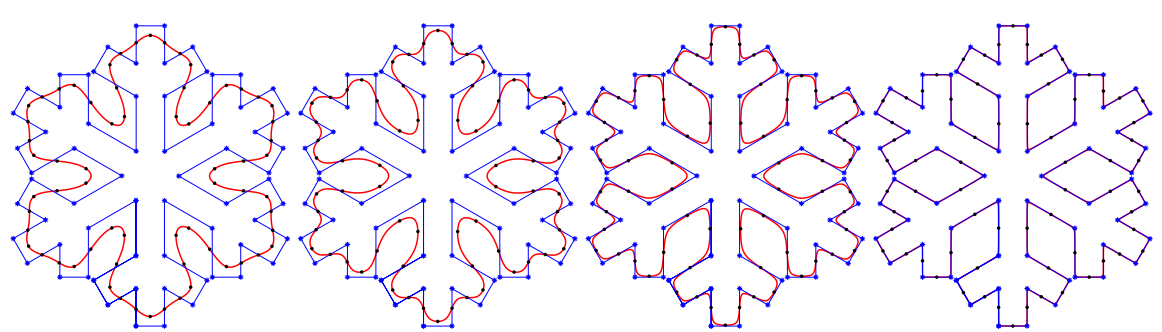

Fig. $10 C^{5}$ splines, knot spacing $h=3$, all pieces taken from the space spanned by $1, \cosh (a x) \cos x$, $\cosh (a x) \sin x, \sinh (a x) \cos x, \sinh (a x) \sin x, \cosh (a x), \sinh (a x)$. From left to right: $a=0.01 ; 0.7 ; 1.5 ; 3$.

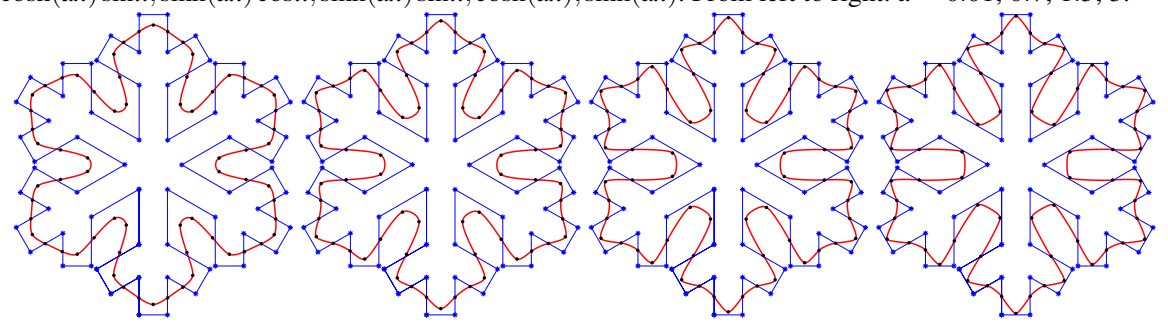

Fig. $11 C^{5}$ splines, knot spacing $h=3$, all pieces taken from the space spanned by $1, \cosh (a x) \cos x$, $\cosh (a x) \sin x, \sinh (a x) \cos x, \sinh (a x) \sin x, \cos x, \sin x$. From left to right: $a=0.01 ; 0.7 ; 1.5 ; 3$.

1- those resulting from the non-real cases of dimension five after double integration steps;

2- spaces spanned by $1, \cos (a x), \sin (a x), \cos (b x), \sin (b x), \cos (c x), \sin (c x)$, where $a, b, c$ are pairwise distinct positive parameters;

3 - spaces spanned by $1, \cosh (a x) \cos (b x), \cosh (a x) \sin (b x), \sinh (a x) \cos (b x)$, $\sinh (a x) \sin (b x), \cosh (c x), \sinh (c x)$;

4- spaces spanned by $1, \cosh (a x) \cos (b x), \cosh (a x) \sin (b x), \sinh (a x) \cos (b x)$, $\sinh (a x) \sin (b x), \cos (c x), \sin (c x)$.

This numerical checking is a very little step towards a possible proof of the conjecture (42), but no doubt that this confirmation is interesting to obtain. The third category is illustrated in Fig. 10 with $a=c, b=1$, and the fourth one in Fig. 11 with $b=c=1$, and in both cases with $h=3$.

\section{Conclusion}

Beyond the classical polynomial splines, the huge class of PEC-splines contains many famous interesting subclasses such as L-splines, geometrically continuous polynomial splines, Müntz splines, ... As a rough statement, all that can be done with classical splines, can also be done with PEC-splines good for design/interpolation, the advantage being that it can be done with a large variety of shape parameters (attached either to the section-spaces or to the connection matrices). If the many examples in the literature clearly demonstrate this advantage, the price to pay for it is the greater difficulty to handle such splines. In many situations, it is not even expectable 
to easily obtain an exact answer to the question: is this PEC-spline space good for design/interpolation? The numerical test was precisely developed for this reason, with a view to facilitate not only the access to a greater number of appropriate PEC-splines but also their use for any kind of applications. To cite only one example, the generalised splines successfully introduced in Isogeometric Analysis [5,6] constitute only a very small subclass of PEC-splines good for design.

Though no numerical characterisation can play the same role as general exact characterisations (as were obtained in [33], for instance), we believe that the few situations that we selected to illustrate it give a clear idea of the usefulness of this test. We would like to insist that our test and its implementation are extremely general, and applicable to any given PEC-spline space, whatever the knot-vector, the multiplicities, the connection matrices, and the EC-section-spaces can be. Nevertheless, replacing the classical splines by PEC-splines is justified only if we are able to handle the shape parameters such splines provide. This is why in practice it is necessary to drastically limitate their numbers. It is worthwhile mentioning that the test is also applicable in the larger context of PQEC-splines (Piecewise Quasi Extended Chebyshev splines, see [31] and other references therein), considering that the class of all PQEC-splines good for design can be understood as the largest class satisfying all properties expected for design. Icing on the cake, our test can provide valuable help on the theoretical side by the fact that it gives access to higher dimensions: it can consolidate conjectures, as in Subsection 4.3, or even suggest some new ones, see Remark 4.

\section{References}

1. Barry P. J., de Boor-Fix dual functionals and algorithms for Tchebycheffian B-splines curves, Constr. Approx., 12 (1996), 385-408.

2. C. Beccari, G. Casciola, and M.-L. Mazure, Piecewise Extended Chebyshev spaces: A numerical test for design, Applied Math. Comput., 296 (2017), 239-256.

3. D. Bister and H. Prautzsch, A New Approach to Tchebycheffian B-Splines in Curves and Surfaces with Applications in CAGD, Vanderbilt University Press, 35-41, 1997.

4. T. Bosner, Knot insertion algorithms for Chebyshev splines, PhD thesis, Dept of Maths, University of Zagreb, 2006.

5. C. Bracco, T. Lyche, C. Manni, F. Roman, H. Speleers, Generalized spline spaces over T-meshes: Dimension formula and locally refined generalized B-splines, Applied Math. Comput., 272 (2016), 187-198.

6. C. Bracco, T. Lyche, C. Manni, F. Roman, H. Speleers, On the dimension of Tchebycheffian spline spaces over planar T-meshes, Comput. Aided Geom. Design, 45 (2016), 151-173.

7. Brilleaud M. and M.-L. Mazure, Mixed hyperbolic/trigonometric spaces for design, Comput. Math. Appl., 64 (2012), 2459-2477.

8. Brilleaud M. and M.-L. Mazure, Design with L-splines, Numer. Algorithms, 65 (2014), 91-124.

9. Carnicer J.-M. and J.-M. Peña, Total positivity and optimal bases, in: M. Gasca, C. A. Micchelli (eds.) Total Positivity and its Applications, Math. Appl. 359, Klüwer Academic Publishers, Dordrecht, 133$155,1996$.

10. J.-M. Carnicer, E. Mainar, and J.-M. Peña, Critical Length for Design Purposes and Extended Chebyshev Spaces, Constr. Approx., 20 (2004), 55-71.

11. P. Costantini, T. Lyche, and C. Manni, On a class of weak Tchebycheff systems, Numer. Math., 101 (2005), 333-354.

12. N. Dyn and C.A. Micchelli, Piecewise polynomial spaces and geometric continuity of curves, Num. Math., 54 (1988), 319-337.

13. T.N.T. Goodman, Properties of beta-splines, J. Approx. Theory, 44 (1985), 132-153. 
14. T.N.T. Goodman, Total Positivity and the Shape of Curves, M. Gasca, C. A. Micchelli (eds.) Total Positivity and its Applications, Math. Appl. 359, Klüwer Academic Publishers, Dordrecht, 157-186, 1996.

15. S. J. Karlin and W. J. Studden, Tchebycheff Systems: with applications in analysis and statistics, Wiley Interscience, New York, 1966.

16. P.-J. Laurent, M.-L. Mazure, and V. T. Maxim, Chebyshev splines and shape parameters, Num. Algorithms, 15 (1997), 373-383.

17. P.-J. Laurent and P. Sablonnière, Pierre Bézier: An engineer and a mathematician, Comput. Aided Geom. Design., 18 (2001), 609-617.

18. T. Lyche, A recurrence relation for Chebyshevian B-splines, Constr. Approx., 1 (1985), 155-178.

19. T. Lyche and L. L. Schumaker, Total Positivity Properties of LB-splines, in Total Positivity and its Applications, M. Gasca and C. Micchelli (eds.), Klüwer, Dordrecht, 35-46, 1996.

20. M.-L. Mazure, Blossoming: a geometrical approach, Constr. Approx., 15 (1999), 33-68.

21. M.-L. Mazure, Chebyshev splines beyond total positivity, Adv. Comput. Math., 14 (2001), 129-156.

22. M.-L. Mazure, Blossoms and optimal bases, Adv. Comp. Math., 20 (2004), 177-203.

23. M.-L. Mazure, On the equivalence between existence of B-spline bases and existence of blossoms, Constr. Approx., 20 (2004), 603-624.

24. M.-L. Mazure, Chebyshev spaces and Bernstein bases, Constr. Approx., 22 (2005), 347-363.

25. M.-L. Mazure, Ready-to-blossom bases in Chebyshev spaces, in: K. Jetter, M. Buhmann, W. Haussmann, R. Schaback, J. Stoeckler (eds.) Topics in Multivariate Approximation and Interpolation, Elsevier, Amsterdam, 109-148, 2006.

26. M.-L. Mazure, A few remarks on recurrence relations for geometrically continuous piecewise Chebyshevian B-splines, Numer. Algorithms, 51 (4), 401-428, 2009.

27. M.-L. Mazure, Finding all systems of weight functions associated with a given Extended Chebyshev space, J. Approx. Theory, 163 (2011), 363-376.

28. M.-L. Mazure, How to build all Chebyshevian spline spaces good for geometric design?, Numer. Math., 119 (2011), 517-556.

29. M.-L. Mazure, Polynomial splines as examples of Chebyshevian splines, Numer. Algorithms, 60 (2012), 241-262.

30. M.-L. Mazure, From Taylor interpolation to Hermite interpolation via duality, Jaén J. Approx., 4 (2012), 15-45.

31. M.-L. Mazure, NURBS or not NURBS, C. R. Acad. Sci. Paris, Ser. I, 354 (2016) 747-750.

32. M.-L. Mazure, Piecewise Chebyshevian splines: Interpolation versus Design, Numer. Algorithms, DOI 10.1007/s11075-017-0360-7.

33. M.-L. Mazure, Constructing totally positive piecewise Chebyshevian B-spline bases, submitted, hal01587487.

34. G. Mühlbach, One sided Hermite interpolation by piecewise different generalized polynomials, J. Comput. Applied Math., 196 (2006), 285-298.

35. H. Pottmann, The geometry of Tchebycheffian splines, Comput. Aided Geom. Design, 10 (1993), $181-210$.

36. L. Ramshaw, Blossoms are polar forms, Comput. Aided Geom. Design, 6 (1989), 323-358.

37. Schumaker L.L., Spline Functions, Wiley Interscience, New York, 1981. 